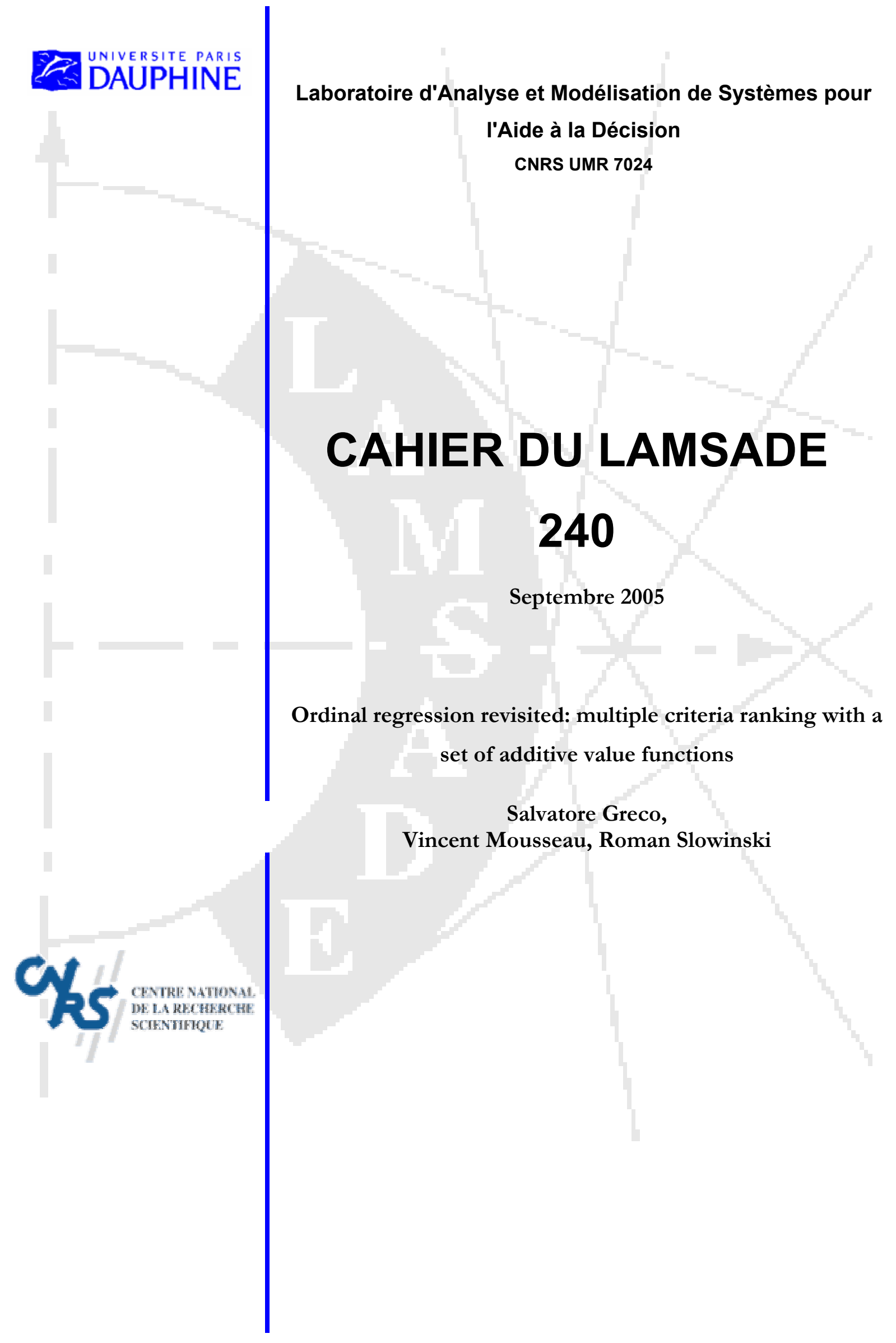




\title{
Ordinal regression revisited: multiple criteria ranking with a set of additive value functions
}

\author{
Salvatore Greco*, Vincent Mousseau ${ }^{\dagger}$ Roman Słowiński ${ }^{\ddagger}$
}

September 9, 2005

\footnotetext{
*Faculty of Economics, University of Catania, Corso Italia, 55, 95129 Catania, Italy, e-mail: salgreco@mbox.unict.it

${ }^{\dagger}$ LAMSADE, Université Paris Dauphine, Place du Maréchal De Lattre de Tassigny, 75775 Paris Cedex 16, France, e-mail: mousseau@lamsade.dauphine.fr

${ }^{\ddagger}$ Institute of Computing Science, Poznań University of Technology, 60-965 Poznań, and Institute for Systems Research, Polish Academy of Sciences, 01-447 Warsaw, Poland, e-mail: roman.slowinski@cs.put.poznan.pl
} 


\section{Contents}

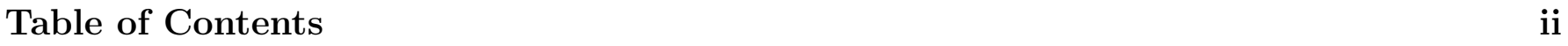

$\begin{array}{ll}\text { Abstract } & \text { iii }\end{array}$

1 Introduction $r$

2 Ordinal regression via linear programming - principle of the UTA method 2

3 Existing approaches and motivations for a new method 5

4 The new UTA ${ }^{\text {GMS }}$ method $\quad 6$

5 Extensions $r \begin{array}{lr}17\end{array}$

5.1 Specification of pairwise comparisons with gradual confidence levels . . . . . . . . . 17

5.2 Accounting for ordinal intensity of preference . . . . . . . . . . . . . . . 20

6 Illustrative example $r$

7 Conclusion $r$ 


\section{Abstract}

We present a new method (called UTA ${ }^{\text {GMS }}$ ) for multiple criteria ranking using strongly and weakly established weak preference relations which result from an ordinal regression. The preference information supplied by the decision maker is a set of pairwise comparisons of reference alternatives. The preference model built via ordinal regression is a set of general additive value functions. The method provides two final rankings: a strong ranking identifying "sure" preference statements, and a weak ranking identifying "possible" preference statements. In order to build these two rankings, the method takes into account all value functions compatible with the preference information. The UTA GMS method is intended to be used interactively, with progressive statement of pairwise comparisons. Moreover, the method can support the decision maker also when his/her preference statements cannot be represented in terms of an additive value function. The method is illustrated by an example solved using the $\mathrm{UTA}^{\mathrm{GMS}}$ software. Some extensions of the method are also presented.

Keywords: Multiple criteria ranking, Ordinal regression approach, Additive value function. 


\section{Introduction}

We are considering a decision situation in which a finite set of alternatives (actions) $A$ is evaluated on a family of $n$ criteria $g_{1}, g_{2}, \ldots, g_{i}, \ldots, g_{n}$, with $g_{i}: A \rightarrow \mathbb{R}$ for all $i \in G=\{1,2, \ldots, n\}$. We assume, without loss of generality, that the greater $g_{i}(a)$, the better alternative $a$ on criterion $g_{i}$, for all $i \in G$. A decision maker (DM) is willing to rank the alternatives in $A$ from the best to the worst, according to his/her preferences. The ranking can be complete or partial, depending on the preference information supplied by the DM and on the way of exploiting this information. The family of criteria $G$ is supposed to satisfy following consistency conditions (see [19]):

- exhaustivity - any two alternatives having the same evaluations on all criteria from $G$ should be considered indifferent,

- monotonicity - when comparing two alternatives, an improvement of one of them on at least one criterion from $G$ should not deteriorate its comparison to the other alternative,

- non-redundancy - deletion of any criterion from $G$ will contradict one of the two above conditions.

Such a decision problem is called multiple criteria ranking problem. It is known that the only information coming out from the formulation of this problem is the dominance ranking. Let us recall that in the dominance ranking, alternative $a \in A$ is preferred to alternative $b \in A$ (denotation $a \succ b$ ) if and only if $g_{i}(a) \geq g_{i}(b)$ for all $i \in G$, with at least one strict inequality; moreover, $a$ is indifferent to $b$ (denotation $a \sim b$ ) if and only if $g_{i}(a)=g_{i}(b)$ for all $i \in G$; hence, for any two alternatives $a, b \in A$, one of the four situations may arise in the dominance ranking: $a \succ b, b \prec a, a \sim b$ and $a ? b$, where the last one means that $a$ and $b$ are incomparable. Usually, the dominance ranking is very poor, i.e. the most frequent situation is $a ? b$.

In order to enrich the dominance ranking, multiple criteria decision aiding (MCDA) helps in construction of an aggregation model on the base of preference information supplied by the DM. Such an aggregation model is called preference model - it induces a preference structure in set $A$ whose proper exploitation permits to work out a ranking proposed to the DM.

The preference information may be either direct or indirect, depending if it specifies directly values of some parameters used in the preference model (e.g. trade-off weights, aspiration levels, discrimination thresholds, etc.), or if it specifies some examples of holistic judgments from which compatible values of the preference model parameters are induced. Direct preference information is used in the traditional aggregation paradigm, according to which the aggregation model is first constructed and then applied on set $A$ to rank the alternatives.

Indirect preference information is used in the disaggregation (or regression) paradigm, according to which the holistic preferences on a subset of alternatives $A^{R} \subseteq A$ are known first and then a consistent aggregation model is inferred from this information to be applied on set $A$ in order to rank the alternatives.

Presently, MCDA methods based on indirect preference information and the disaggregation paradigm are of increasing interest for they require relatively less cognitive effort from the DM. Indeed, the disaggregation paradigm is consistent with the "posterior rationality" postulated by March [13] and with the inductive learning used in artificial intelligence approaches (see [14]). Typical applications of this paradigm in MCDA are presented in [23], [17], [7], [9], [1], [16], [4], [5], [6]. In this 
paper, we are considering the aggregation model in form of an additive value function:

$$
U(a)=\sum_{i=1}^{n} u_{i}(a)
$$

where $u_{i}(a) \geq 0, i=1, \ldots, n$, are nondecreasing marginal value functions. We are using this aggregation model in the settings of the disaggregation paradigm, as it has been proposed in the UTA method (see [7]). In fact, our method generalizes the UTA method by considering the set of all additive value functions (1) compatible with indirect preference information having the form of a set of pairwise comparisons of some reference alternatives $A^{R} \subseteq A$. As a result, we will obtain two rankings in the set of alternatives $A$, such that for any pair of alternatives $a, b \in A$ :

- in the first (strong) ranking, $a$ is ranked at least as good as $b$ if and only if, $U(a) \geq U(b)$ for all value functions compatible with the indirect preference information,

- in the second (weak) ranking, $a$ is ranked at least as good as $b$ if and only if, $U(a) \geq U(b)$ for at least one value function compatible with the indirect preference information.

The first (strong) ranking can be considered as robust with respect to the indirect preference information. Such robustness of the strong ranking refers to the fact that any pair of alternatives compares in the same way whatever the additive value function compatible with the indirect preference information. Indeed, when no indirect preference information is given, the strong ranking corresponds to the dominance ranking.

Another appeal of such an approach stems from the fact that it gives space for interactivity with the DM. Considering an indirect preference information provided by the DM, the presentation of the strong ranking is a good support for generating reactions from the DM. Namely, (s)he could wish to enrich the ranking or to contradict a part of it. This reaction would be integrated in the indirect preference information in the next iteration.

The organization of the paper is the following. In the next section, we will outline the principle of the ordinal regression via linear programming, as proposed in the original UTA method (see [7]). In section 3, we give a brief overview of existing approaches to multiple criteria ranking with a set of additive value functions, and we provide motivations for our approach. The new UTA GMS method is presented in section 4 . The illustrative example provided in section 6 shows how the method can be applied in practice. Some extensions are considered in section 5 and the last section includes conclusions.

\section{Ordinal regression via linear programming - principle of the UTA method}

Let $X_{i}$ denote the domain of criterion $g_{i}, i \in G$. Consequently, $X=\prod_{i=1}^{n} X_{i}$ is the evaluation space, and $x, y \in X$ denote profiles of alternatives in this space. We consider an outranking (weak preference) relation $\succsim$ on $X$ that states for each pair of vectors $x, y \in X: x \succsim y \Leftrightarrow$ " $x$ is at least as good as $y "$. This outranking relation can be decomposed into its asymmetric and symmetric parts, as follows:

- $x \succ y \Leftrightarrow[x \succsim y$ and $\operatorname{not}(y \succsim x)] \Leftrightarrow " x$ is preferred to $y "$,

- $x \sim y \Leftrightarrow[x \succsim y$ and $y \succsim x] \Leftrightarrow " x$ is indifferent to $y "$ 
From a pragmatic viewpoint, it is reasonable to assume that $X_{i} \subset \mathbb{R}$, for all $i=1, \ldots, n$. More specifically, we will assume that the evaluation scale on each criterion $g_{i}$ is bounded, such that $X_{i}=\left[\alpha_{i}, \beta_{i}\right]$ where $\alpha_{i}<\beta_{i}$ are the worst and the best (finite) evaluations, respectively. Thus, $g_{i}: A \mapsto X_{i}, i \in G$, therefore, each alternative $a \in A$ is associated with an evaluation vector denoted by $\underline{g}(a) \in X$.

The additive value function is defined on $X$ such that for each $a \in A$

$$
U(\underline{g}(a))=\sum_{i=1}^{n} u_{i}\left(g_{i}(a)\right)
$$

where $u_{i}$ are non-decreasing marginal value functions, $u_{i}: X_{i} \mapsto \mathbb{R}, i=1, \ldots, n$. For simplicity, we will write $(2)$ as $(1)$, i.e. $U(a)=\sum_{i=1}^{n} u_{i}(a)$.

In the following, we present the principle of the UTA method as presented recently in [22]. The indirect preference information is given in form of a complete preorder on a subset of reference alternatives $A^{R} \subseteq A$, called reference preorder. The reference alternatives are usually those alternatives in set $A$ for which the DM is ready to express holistic preferences. Let the set of reference alternatives $A^{R}=\left\{a_{1}, a_{2}, \ldots, a_{m}\right\}$ be rearranged such that $a_{k} \succsim a_{k+1}, k=1, \ldots, m-1$, where $m=\left|A^{R}\right|$. The disaggregation paradigm consists here in inferring an additive value function (1) compatible with this reference preorder. The inferred value of each reference alternative $a \in A^{R}$ is:

$$
U^{\prime}(a)=\sum_{i=1}^{n} u_{i}(a)+\sigma^{+}(a)-\sigma^{-}(a)
$$

where $\sigma^{+}(a)$ and $\sigma^{-}(a), \sigma^{+}(a) \geq 0$ and $\sigma^{-}(a) \geq 0$ for all $a \in A^{R}$, are potential errors of overand under-estimation of the "correct" value $U(a)=\sum_{i=1}^{n} u_{i}(a)$, respectively. In the UTA method, the marginal value functions $u_{i}$ are assumed to be piecewise linear, so that the intervals $\left[\alpha_{i}, \beta_{i}\right]$ are divided into $\gamma_{i} \geq 1$ equal sub-intervals: $\left[x_{i}^{0}, x_{i}^{1}\right],\left[x_{i}^{1}, x_{i}^{2}\right], \ldots,\left[x_{i}^{\gamma_{i}-1}, x_{i}^{\gamma_{i}}\right]$, where $x_{i}^{j}=\alpha_{i}+\frac{j\left(\beta_{i}-\alpha_{i}\right)}{\gamma_{i}}$, $j=0, \ldots, \gamma_{i}, i=1, \ldots, n$. The marginal value (see Figure 1 ) of an alternative $a \in A$ is approximated by linear interpolation

$$
u_{i}(a)=u_{i}\left(x_{i}^{j}\right)+\frac{g_{i}(a)-x_{i}^{j}}{x_{i}^{j+1}-x_{i}^{j}}\left(u_{i}\left(x_{i}^{j+1}\right)-u_{i}\left(x_{i}^{j}\right)\right), \text { for } g_{i}(a) \in\left[x_{i}^{j}, x_{i}^{j+1}\right]
$$

According to (4), the piecewise linear additive model is completely defined by the marginal values at the breakpoints, i.e. $u_{i}\left(x_{i}^{0}\right)=u_{i}\left(\alpha_{i}\right), u_{i}\left(x_{i}^{1}\right), u_{i}\left(x_{i}^{2}\right), \ldots, u_{i}\left(x_{i}^{\gamma_{i}}\right)=u_{i}\left(\beta_{i}\right)$.

The ordinal regression consists in the inference of a value function restoring the reference preorder. We call compatible any value function obtained by ordinal regression. The transition from a reference preorder to a value function is done according to the following equivalence :

$$
\begin{aligned}
& U^{\prime}\left(a_{k}\right)>U^{\prime}\left(a_{k+1}\right) \Leftrightarrow a_{k} \succ a_{k+1} \\
& U^{\prime}\left(a_{k}\right)=U^{\prime}\left(a_{k+1}\right) \Leftrightarrow a_{k} \sim a_{k+1}
\end{aligned}
$$

for $k=1, \ldots, m-1$.

Let us remark that the transition from the preorder to the marginal value function exploits the ordinal character of the criterion scale $X_{i}$ even if the scale is richer (interval or ratio scale). Note, however, that the scale of the marginal value function is an interval scale and thus its nature is cardinal. 


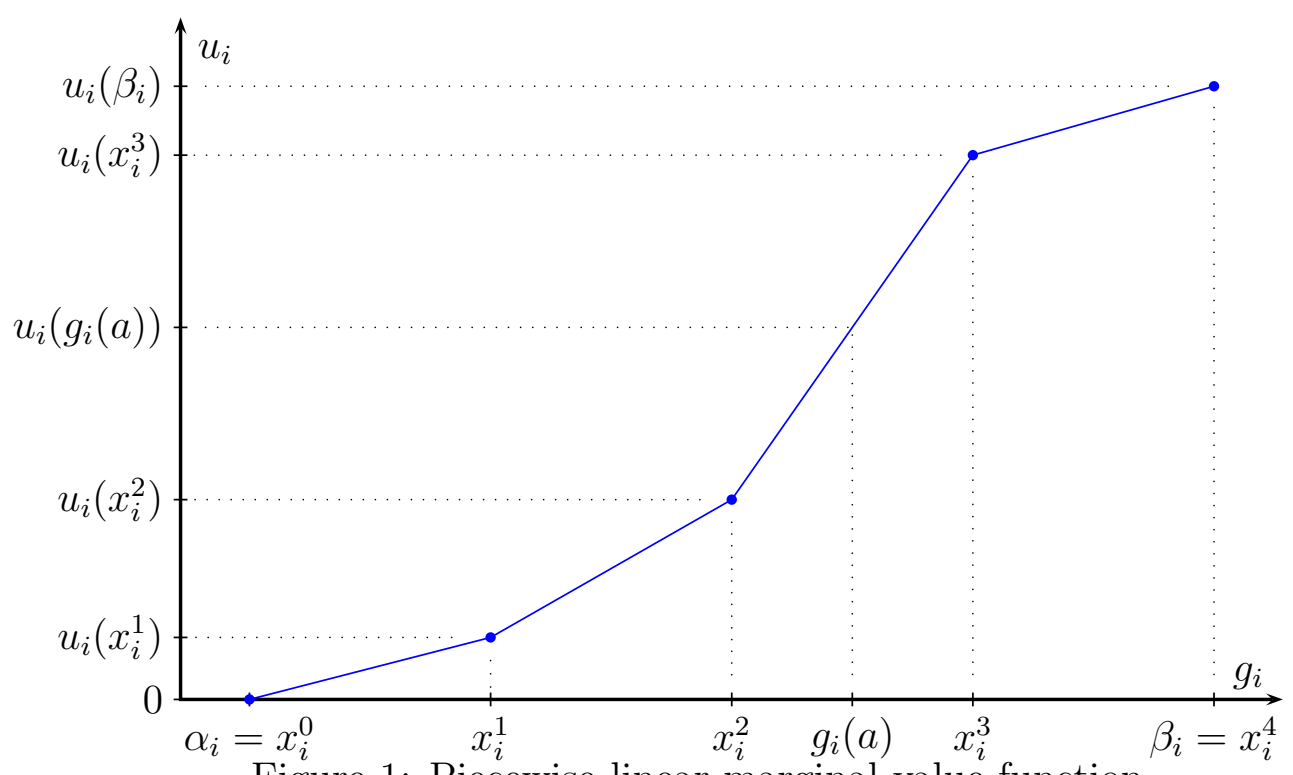

Figure 1: Piecewise linear marginal value function

The above transition requires, moreover, the marginal value functions to be monotonic with respect to each criterion $g_{i}, \forall i \in G$, i.e.:

$$
u_{i}\left(x_{i}^{j+1}\right)-u_{i}\left(x_{i}^{j}\right) \geq 0, j=0, \ldots, \gamma_{i}-1, i=1, \ldots, n
$$

It is also usual to suppose a kind of normalization such as $u_{i}\left(\alpha_{i}\right)=0 \quad \forall i \in G$, and $\sum_{i=1}^{n} u_{i}\left(\beta_{i}\right)=1$. This will bound the value function $U(a)$ in the interval $[0,1]$.

An alternative way of representing the same preference model is:

$$
U(a)=\sum_{i=1}^{n} w_{i} \hat{u}_{i}(a), \quad \text { where } \hat{u}\left(\alpha_{i}\right)=0, \hat{u}\left(\beta_{i}\right)=1, w_{i} \geq 0 \quad \forall i \in G, \text { and } \sum_{i=1}^{n} w_{i}=1
$$

Note that the correspondence between (7) and (1) is such that $w_{i}=u_{i}\left(\beta_{i}\right), \forall i \in G$. Due to the cardinal character of the marginal value function scale, the parameters $w_{i}$ can be interpreted as tradeoff weights among marginal value functions $\hat{u}_{i}(a)$. We will use, however, the preference model (1) with normalization constraints bounding $U(a)$ to the interval $[0,1]$.

Consequently, the piecewise linear additive value model is inferred through the resolution of the following mathematical program, called ordinal regression problem (8):

$$
\left.\begin{array}{rl}
\text { Min } \rightarrow \quad F=\sum_{a \in A^{R}}\left(\sigma^{+}(a)+\sigma^{-}(a)\right) \\
\text { s.t. } \\
& \left.U^{\prime}\left(a_{k}\right)>U^{\prime}\left(a_{k+1}\right) \Leftrightarrow a_{k} \succ a_{k+1}\right\} k=1, \ldots, m-1 \\
& U^{\prime}\left(a_{k}\right)=U^{\prime}\left(a_{k+1}\right) \Leftrightarrow a_{k} \sim a_{k+1} \\
& u_{i}\left(x_{i}^{j+1}\right)-u_{i}\left(x_{i}^{j}\right) \geq 0, i=1, \ldots, n, j=1, \ldots, \gamma_{i}-1 \\
& u_{i}\left(\alpha_{i}\right)=0, i=1, \ldots, n \\
& \sum_{i=1}^{n} u_{i}\left(\beta_{i}\right)=1 \\
& \sigma^{+}\left(a_{k}\right), \sigma^{-}\left(a_{k}\right) \geq 0, k=1, \ldots, m
\end{array}\right\}
$$

Remark that (8) is a linear program if the first constraint $U^{\prime}\left(a_{k}\right)>U^{\prime}\left(a_{k+1}\right)$ is rewritten as $U^{\prime}\left(a_{k}\right) \geq U^{\prime}\left(a_{k+1}\right)+\varepsilon$ where $\varepsilon$ is an arbitrarily small positive value. 
If the optimal value of the objective function of the program $(8)$ is equal to zero $\left(F^{*}=0\right)$, then there exists at least one value function $U^{\prime}(a)=U(a)$, compatible with the reference preorder on $A^{R}$ which means that the corresponding polyhedron of feasible solutions for $u_{i}\left(x_{i}^{j}\right), i=1, \ldots, n, j=$ $1, \ldots, \gamma_{i}$ is not empty.

When the optimal value of the objective function of the program $(8)$ is greater than zero $\left(F^{*}>0\right)$, then there is no value function $U(a)$ compatible with the reference preorder on $A^{R}$. In such a case, four possible moves can be considered:

- accepting the value function inferred even if it does not represent perfectly the DM's preferences,

- increasing the number of linear pieces $\gamma_{i}$ for one or several marginal value function $u_{i}$ : this could make it possible to find an additive value function compatible with the reference preorder on $A^{R}$,

- revising the reference preorder on $A^{R}$ : this could lead to find an additive value function compatible with the new preorder,

- searching over the relaxed domain $F \leq F^{*}+\eta$ : this could lead to an additive value functions giving a preorder on $A^{R}$ sufficiently close to the reference preorder (in the sense of Kendall's $\tau)$.

\section{Existing approaches and motivations for a new method}

Our work aims at generalizing the UTA method in order to consider the set of all utility functions compatible with the indirect preference information rather than choosing a single utility function within the set of compatible ones. The literature concerning MCDA methods involving a set of additive value functions can be viewed from three points of view:

- The methods are designed for different problem statements (problematics, see [18]):

- choice of the best alternative (e.g. [2], [10], [20]),

- sorting alternatives into predefined categories (e.g. [11], [3]),

- ranking of alternatives from the best to the worst $(e . g .[7],[8])$

- The methods also differ with respect to the kind of the set of value functions and the characteristics of these functions: linear (e.g. [10], [8]) or piecewise linear (e.g. [7], [3]) or monotonic (e.g. [1]) value functions.

- The sets of value functions can be:

- explicitly listed (e.g. [21]),

- defined from stated constraints on the functions (e.g. [2], [12]),

- induced from holistic preference statements concerning alternatives (e.g. [11], [24], [1]).

A review of the literature and, particularly, of the methods based on the ordinal regression approach, shows that these methods fail to consider some important issues : 
- If the polyhedron of value functions compatible with the stated preference information is not empty, then the choice of a single or few representative value functions is either arbitrary or left to the DM. In the latter case, the DM is supposed to know how to interpret the form of the marginal value functions in order to choose among them, which is not easy for most DMs. Therefore, it seems reasonable to accept existence of all value functions compatible with the preference information provided by the DM and to assess a preference relation in the set of alternatives $A$ with respect to all these functions.

- In most methods, the class of value functions is limited to piecewise linear marginal value functions. To specify the number of breakpoints is arbitrary and restrictive. It is desirable to consider just monotonic marginal value functions which do not involve any parametrization.

- Most methods require that the DM provides constraints on the range of "weights" or on the range of marginal value functions. The DM may have, however, difficulties to analyze the link between a specific value function and the resulting ranking. This is why we believe that the DM should be allowed to express preference information in terms of pairwise comparisons of alternatives rather than fixing the above constraints. Providing preference information in this way is consistent with intuitive reasoning of DMs.

- The methods based on ordinal regression are usually considering the preference information provided by the DM as a whole. As a consequence, it is difficult for the DM to associate a piece of his/her preference information with the result and, therefore, to control the impact of each piece of information (s)he provides on the result. As such a control is desirable for a truly interactive process, ordinal regression methods should allow the DM to provide incrementally the preference information by possibly small pieces.

In this paper, we intend to present a new ordinal regression method that accounts for all shortcomings listed above.

\section{The new UTA ${ }^{\mathrm{GMS}}$ method}

The new UTA ${ }^{\text {GMS }}$ method is another ordinal regression method using a set of additive value functions $U(a)=\sum_{i=1}^{n} u_{i}\left[g_{i}(a)\right]$ as a preference model. One of its characteristic features is that it takes into account the set of all value functions compatible with the preference information provided by the DM. Moreover, it considers any monotonic marginal value function instead of piecewise linear only.

We suppose the DM provides preference information in form of pairwise comparisons of reference alternatives from $A^{R} \subseteq A$ (a preorder, not necessarily complete). By $B^{R} \subseteq A^{R} \times A^{R}$ we denote the set of pairs of reference alternatives compared by the DM. A value function is called compatible if it is able to restore all pairwise comparisons from $B^{R}$. Each compatible value function induces, moreover, a ranking on the whole set $A$.

In particular, for any two alternatives $x, y \in A$, a compatible value function ranks $x$ and $y$ in one of the following ways: $x \succ y, y \succ x, x \sim y$. With respect to $x, y \in A$, it is thus reasonable to ask the following two questions:

- are $x$ and $y$ ranked in the same way by ALL compatible value functions?

- is there AT LEAST ONE compatible value function ranking $x$ at least as good as $y$ (or $y$ at least as good as $x)$ ? 
Having answers to these questions for all pairs of alternatives $(x, y) \in A \times A$, one gets a strongly established weak preference relation $\succsim^{S}$, whose semantics is that for all compatible value functions $U(x) \geq U(y)$, and a weakly established weak preference relation $\succsim^{W}$ in $A$, whose semantics is that for at least one compatible value functions $U(x) \geq U(y)$.

Let us remark that preference relation $\succsim^{S}$ and $\succsim^{W}$ are meaningful only if there is at least one compatible value function.

Observe also that in this case for any $(x, y) \in B^{R}$

$$
x \succsim y \Rightarrow x \succsim^{S} y
$$

and

$$
x \succ y \Rightarrow \operatorname{not} y \succsim^{W} x .
$$

In fact, as $x \succsim y$, for any compatible value function $U(x) \geq U(y)$, and therefore $x \succsim^{S} y$. Moreover, as $x \succ y$, for any compatible value function $U(x)>U(y)$; consequently there is no compatible value function such that $U(y) \geq U(x)$ and therefore not $y \succsim^{W} x$.

$\succsim^{S}$ and $\succsim^{W}$ will constitute basic results of the $\mathrm{UTA}^{\mathrm{GMS}}$ method.

In order to consider general additive compatible value functions, we propose to fix the breakpoints of marginal value functions $u_{i}\left(g_{i}\right), i=1, \ldots, n$, in points corresponding to evaluations on criterion $g_{i}$ of all reference alternatives and of the two other considered alternatives $x, y \notin B^{R}$. Let $\pi_{i}$ be a permutation on the set of alternatives $A^{R} \cup\{x, y\}$ that reorders them according to increasing evaluation on criterion $g_{i}$, i.e.

$$
g_{i}\left(a_{\pi_{i}(1)}\right) \leq g_{i}\left(a_{\pi_{i}(2)}\right) \leq \ldots \leq g_{i}\left(a_{\pi_{i}(\omega-1)}\right) \leq g_{i}\left(a_{\pi_{i}(\omega)}\right)
$$

where

- if $A^{R} \cap\{x, y\}=\emptyset$, then

$$
\omega=m+2
$$

- if $A^{R} \cap\{x, y\}=\{x\}$ or $A^{R} \cap\{x, y\}=\{y\}$, then

$$
\omega=m+1
$$

- if $A^{R} \cap\{x, y\}=\{x, y\}$, then

$$
\omega=m \text {. }
$$

Then, we can fix the breakpoints of $u_{i}\left(g_{i}\right), i=1, \ldots, n$, in

$$
g_{i}^{0}=\alpha_{i}, \quad g_{i}^{j}=g_{i}\left(a_{\pi_{i}(j)}\right) \text { for } j=1, \ldots, \omega, \quad g_{i}^{\omega+1}=\beta_{i}
$$

Remark that, due to this definition of the breakpoints, no linear interpolation is required to express the marginal value of any reference alternative considered in the ordinal regression problem (8). Thus, one cannot expect that increasing the number of breakpoints will bring some "new" compatible additive value functions. In consequence, fixing the breakpoints in this way ensures that we consider all compatible additive value functions. 
For any pair of alternatives $x, y \in A$ and for the available preference information about comparisons of pairs of reference alternatives from set $B^{R}$, preference with respect to $x$ and $y$ is determined by compatible value functions, that is functions $U$ verifying the following set $E(x, y)$ of ordinal regression constraints:

$$
\begin{aligned}
& U^{\prime}(a)>U^{\prime}(b) \Leftrightarrow a \succ b \\
& U^{\prime}(a)=U^{\prime}(b) \Leftrightarrow a \sim b \\
& u_{i}\left(g_{i}^{j}\right)-u_{i}\left(g_{i}^{j-1}\right) \geq 0, i=1, \ldots, n, j=1, \ldots, \omega+1 \\
& u_{i}\left(g_{i}^{0}\right)=0, i=1, \ldots, n \\
& \sum_{i=1}^{n} u_{i}\left(g_{i}^{\omega+1}\right)=1, \\
& \sigma^{+}(a)=0, \sigma^{-}(a)=0, \forall a \in A^{R}
\end{aligned}
$$

Note that for all $x, y \in A, E(x, y)=E(y, x)$.

Let us suppose that the polyhedron defined by the set of constraints $E(x, y)$ is not empty. In the two following definitions, we define formally a strongly established weak preference relation $\succsim^{S}$ and a weakly established weak preference relation $\succsim^{W}$ on $A$, which were announced above.

Definition 4.1. $\succsim^{S}$ (strongly established weak preference relation) is defined as follows: for all $x, y \in A$

$$
\begin{aligned}
x & \succsim^{S} y \Leftrightarrow d(x, y) \geq 0 \\
d(x, y)= & \operatorname{Min}\{U(x)-U(y)\} \\
& \text { s.t. set } E(x, y) \text { of constraints }
\end{aligned}
$$

where:

Definition 4.2. $\succsim^{W}$ (weakly established weak preference relation) is defined as follows: for all $x, y \in$ A

where :

$$
x \succsim^{W} y \Leftrightarrow D(x, y) \geq 0
$$

$$
\begin{aligned}
D(x, y)= & \operatorname{Max}\{U(x)-U(y)\} \\
& \text { s.t. set } E(x, y) \text { of constraints }
\end{aligned}
$$

The discussion of some properties of the two preference relations $\succsim^{S}$ and $\succsim^{W}$ requires some preliminary results relative to the set of constraints $E(x, y)$.

Let us consider the following two further sets of constraints:

$$
\left.\begin{array}{l}
U^{\prime}(a)>U^{\prime}(b) \Leftrightarrow a \succ b \\
U^{\prime}(a)=U^{\prime}(b) \Leftrightarrow a \sim b \\
u_{i}\left(g_{i}\left(a_{\tau_{i}(j)}\right)\right)-u_{i}\left(g_{i}\left(a_{\tau_{i}(j-1)}\right)\right) \geq 0, i=1, \ldots, n, j=1, \ldots, m \\
u_{i}\left(g_{i}\left(a_{\tau_{i}(1)}\right) \geq 0, u_{i}\left(g_{i}\left(a_{\tau_{i}(m)}\right)\right) \leq u_{i}\left(\beta_{i}\right), i=1, \ldots, n,\right. \\
u_{i}\left(\alpha_{i}\right)=0, i=1, \ldots, n \\
\sum_{i=1}^{n} u_{i}\left(\beta_{i}\right)=1, \\
\sigma^{+}(a)=0, \sigma^{-}(a)=0, \forall a \in A^{R}
\end{array}\right\} \quad\left(E^{A^{R}}\right)
$$

where $\tau_{i}$ is the permutation on the set of alternatives $A^{R}$ that reorders them according to the increasing evaluation on criterion $g_{i}$, i.e.

$$
g_{i}\left(a_{\tau_{i}(1)}\right) \leq g_{i}\left(a_{\tau_{i}(2)}\right) \leq \ldots \leq g_{i}\left(a_{\tau_{i}(m-1)}\right) \leq g_{i}\left(a_{\tau_{i}(m)}\right) ;
$$




$$
\left.\begin{array}{l}
U^{\prime}(a)>U^{\prime}(b) \Leftrightarrow a \succ b \\
U^{\prime}(a)=U^{\prime}(b) \Leftrightarrow a \sim b \\
u_{i}\left(g_{i}\left(a_{\rho_{i}(j)}\right)\right)-u_{i}\left(g_{i}\left(a_{\rho_{i}(j-1)}\right)\right) \geq 0, i=1, \ldots, n, j=1, \ldots, m^{\prime} \\
u_{i}\left(g_{i}\left(a_{\rho_{i}(1)}\right)\right) \geq 0, u_{i}\left(g_{i}\left(a_{\rho_{i}(m)}\right)\right) \leq u_{i}\left(\beta_{i}\right), i=1, \ldots, n, \\
u_{i}\left(\alpha_{i}\right)=0, i=1, \ldots, n \\
\sum_{i=1}^{n} u_{i}\left(\beta_{i}\right)=1, \\
\sigma^{+}(a)=0, \sigma^{-}(a)=0, \forall a \in A^{R}
\end{array}\right\}\left(E^{A}\right)
$$

where $m^{\prime}=|A|$ and $\rho_{i}$ is the permutation on the set of alternatives $A$ that reorders them according to the increasing evaluation on criterion $g_{i}$, i.e.

$$
g_{i}\left(a_{\rho_{i}(1)}\right) \leq g_{i}\left(a_{\rho_{i}(2)}\right) \leq \ldots \leq g_{i}\left(a_{\rho_{i}\left(m^{\prime}-1\right)}\right) \leq g_{i}\left(a_{\rho_{i}\left(m^{\prime}\right)}\right)
$$

Let us remark that set of constraints $E^{A^{R}}$ is the basic one. In fact, for any pair $(x, y) \in A, E(x, y)$ can be seen as the union of the constraints in $E^{A^{R}}$ with the constraints relative to the breakpoints relative to alternatives $x, y \notin A^{R}$, i.e.

$$
u_{i}\left(g_{i}(x)\right)-\max \left\{u_{i}\left(g_{i}(a)\right): g_{i}(a) \leq g_{i}(x)\right\} \geq 0,
$$

and

$$
\min \left\{u_{i}\left(g_{i}(a)\right): g_{i}(a) \geq g_{i}(x)\right\}-u_{i}\left(g_{i}(x)\right) \geq 0
$$

if $x \notin A^{R}$;

$$
u_{i}\left(g_{i}(y)\right)-\max \left\{u_{i}\left(g_{i}(a)\right): g_{i}(a) \leq g_{i}(y)\right\} \geq 0,
$$

and

$$
\min \left\{u_{i}\left(g_{i}(a)\right): g_{i}(a) \geq g_{i}(y)\right\}-u_{i}\left(g_{i}(y)\right) \geq 0,
$$

if $y \notin A^{R}$.

Analogously, set of constraints $E^{A}$ can be seen as the union of the constraints in $E^{A^{R}}$ with the constraints relative to the break points relative to all alternatives $x \notin A^{R}$. Let us observe that the sets of vectors $\mathbf{u}^{\mathbf{A}}=\left[u_{i}^{A}\left(g_{i}\left(a_{\rho_{i}(j)}\right), i=1, \ldots, n, i=0, \ldots, n, j=1, \ldots, m^{\prime}+1\right]\right.$ satisfying constraint $E^{A}$ gives all the compatible additive value functions on alternatives from $A$.

Proposition 4.1. Consider

$$
\begin{gathered}
\mathbf{u}^{\mathbf{E}(\mathbf{x}, \mathbf{y})}=\left[u_{i}^{E(x, y)}\left(g_{i}^{j}\right), i=1, \ldots, n, j=1, \ldots, \omega+1\right], \\
\mathbf{u}^{\mathbf{A}^{\mathbf{R}}}=\left[u_{i}^{A^{R}}\left(g_{i}\left(a_{\tau_{i}(j)}\right), i=1, \ldots, n, i=0, \ldots, n, j=1, \ldots, m+1\right],\right. \\
\mathbf{u}^{\mathbf{A}}=\left[u_{i}^{A}\left(g_{i}\left(a_{\rho_{i}(j)}\right), i=1, \ldots, n, i=0, \ldots, n, j=1, \ldots, m^{\prime}+1\right]\right.
\end{gathered}
$$

with

$$
g_{i}\left(a_{\tau_{i}(0)}\right)=g_{i}\left(a_{\rho_{i}(0)}\right)=\alpha_{i}
$$

and

$$
g_{i}\left(a_{\tau_{i}(m+1)}\right)=g_{i}\left(a_{\rho_{i}\left(m^{\prime}+1\right)}\right)=\beta_{i} .
$$

Moreover, consider the following conditions: 
1. for all $a \in A^{R}$, if $a=a_{\sigma_{i}(j)}=a_{\tau_{i}(j)}=a_{\rho_{i}(j)}$, then $u_{i}^{E(x, y)}\left(g_{i}^{j}\right)=u_{i}^{A^{R}}\left(g_{i}\left(a_{\tau_{i}(j)}\right)=u_{i}^{A}\left(g_{i}\left(a_{\rho_{i}(j)}\right)\right.\right.$,

2. $u_{i}^{E(x, y)}\left(g_{i}^{1}\right)=u_{i}^{A^{R}}\left(g_{i}\left(a_{\tau_{i}(0)}\right)=u_{i}^{A}\left(g_{i}\left(a_{\rho_{i}(0)}\right)=0, i=1, \ldots, n\right.\right.$

$$
\left(u_{i}^{E(x, y)}\left(g^{(} \alpha_{i}\right)=u_{i}^{A^{R}}\left(g_{i}\left(\alpha_{i}\right)=u_{i}^{A}\left(g_{i}\left(\alpha_{i}\right)=0\right),\right.\right.
$$

3. $u_{i}^{E(x, y)}\left(g^{\omega}+1_{i}\right)=u_{i}^{A^{R}}\left(g_{i}\left(a_{\tau_{i}(m+1)}\right)=u_{i}^{A}\left(g_{i}\left(a_{\rho_{i}\left(m^{\prime}+1\right)}\right), i=1, \ldots, n\right.\right.$

$$
\left(u_{i}^{E(x, y)}\left(g^{(} \beta_{i}\right)=u_{i}^{A^{R}}\left(g_{i}\left(\beta_{i}\right)=u_{i}^{A}\left(g_{i}\left(\beta_{i}\right)=0\right),\right.\right.
$$

4. for all $j=1, \ldots, m^{\prime}+1, u_{i}^{A}\left(g_{i}\left(a_{\rho_{i}(j+1)}\right) \geq u_{i}^{A}\left(g_{i}\left(a_{\rho_{i}(j)}\right)\right.\right.$.

If conditions 1)-4) are satisfied then the following propositions are equivalent:

a) $\mathbf{u}^{\mathbf{A}^{\mathbf{R}}}$ satisfies constraints $E^{A^{R}}$,

b) $\mathbf{u}^{\mathbf{E}(\mathbf{x}, \mathbf{y})}$ satisfies constraints $E(x, y)$,

c) $\mathbf{u}^{\mathbf{A}}$ satisfies constraints $E^{A}$.

Proof: $\mathrm{a}) \Rightarrow \mathrm{b})$. For a) and conditions 1,2 and $3, \mathbf{u}^{\mathbf{E}(\mathbf{x}, \mathbf{y})}$ satisfies constraints in $E^{A^{R}}$. Due to condition 1 and condition 4 , for all $a, b \in A^{R} \cup\{x, y\}$

$$
g_{i}(a) \geq g_{i}(b) \Rightarrow u^{E(x, y)}\left(g_{i}(a)\right) \geq u^{E(x, y)}\left(g_{i}(a)\right)
$$

and therefore $\mathbf{u}^{\mathbf{E}(\mathbf{x}, \mathbf{y})}$ satisfies also all the constraints present in $E(x, y)$ but not in $E^{A^{R}}$.

$\mathrm{b}) \Rightarrow \mathrm{c})$. For b) and conditions 1,2 , and $3, \mathbf{u}^{\mathbf{A}}$ satisfies constraints in $E(x, y)$. For condition 4 , $\mathbf{u}^{\mathbf{A}}$ satisfies also all the constraints present in $E^{A}$ but not in $E(x, y)$.

c) $\Rightarrow$ a). For c) and conditions 1,2 and $3, \mathbf{u}^{\mathbf{A}}$ satisfies constraints in $E^{A^{R}}$.

Proposition 4.2. Consider the following value $d^{A}(x, y)$ and $D^{A}(x, y)$

$$
\begin{aligned}
d^{A}(x, y)= & \operatorname{Min}\{U(x)-U(y)\} \\
& \text { s.t. set } E^{A} \text { of constraints } \\
D^{A}(x, y)= & \operatorname{Max}\{U(x)-U(y)\} \\
& \text { s.t. set } E^{A} \text { of constraints }
\end{aligned}
$$

For all $x, y \in A d^{A}(x, y)=d(x, y)$ and $D^{A}(x, y)=D(x, y)$.

Proof: Given $\mathbf{u}^{\mathbf{A}}$ satisfying set of constraints $E^{A}$ and $\mathbf{u}^{\mathbf{E}(\mathbf{x}, \mathbf{y})}$ satisfying set of constraints $E(x, y)$, we say that they are corresponding if for all $a \in A^{R} \cup\{x, y\}$ and for all $i=1, \ldots, n$

$$
u^{E(x, y)}\left(g_{i}(a)\right)=u^{A}\left(g_{i}(a)\right) .
$$

Let us remark that for each $\mathbf{u}^{\mathbf{A}}$ there is only one corresponding $\mathbf{u}^{\mathbf{E}(\mathbf{x}, \mathbf{y})}$, while, in general, for each $\mathbf{u}^{\mathbf{E}(\mathbf{x}, \mathbf{y})}$ there are infinite corresponding $\mathbf{u}^{\mathbf{A}}$.

We prove that $U(x)-U(y)$ subject to constraints $E^{A}$ reaches its minimum for $\mathbf{u}^{\mathbf{A}}$ if and only if $U(x)-U(y)$ subject to constraints $E(x, y)$ reaches its minimum for the corresponding $\mathbf{u}^{\mathbf{E}(\mathbf{x}, \mathbf{y})}$.

For contraddiction, suppose that $U(x)-U(y)$ subject to constraints $E^{A}$ reaches its minimum in $\mathbf{u}^{\mathbf{A}}$, but $U(x)-U(y)$ subject to constraints $E(x, y)$ reaches its minimum in $\mathbf{u}^{\prime \mathbf{E}(\mathbf{x}, \mathbf{y})}$ which is not corresponding to $\mathbf{u}^{\mathbf{A}}$, i.e. for at least one $a \in A^{R} \cup\{x, y\}$ and for at least one $i=1, \ldots, n$ we have $u^{\prime E(x, y)}\left(g_{i}(a)\right) \neq u^{A}\left(g_{i}(a)\right)$. Two cases are possible: 
- $d^{A}(x, y)>d(x, y)$ : in this case consider $\mathbf{u}^{\prime \mathbf{A}}$ such that for all $i=\ldots n$

$-u^{\prime A}\left(\alpha_{i}\right)=u^{\prime E(x, y)}\left(\alpha_{i}\right)=0, u^{\prime A}\left(\beta_{i}\right)=u^{\prime E(x, y)}\left(\beta_{i}\right)$,

- for all $a \in A^{R} \cup\{x, y\}$

$$
u^{\prime A}\left(g_{i}(a)\right)=u^{\prime E(x, y)}\left(g_{i}(a)\right)
$$

- for all $a \notin A^{R} \cup\{x, y\}$

$$
u^{\prime A}\left(g_{i}(a)\right)=\min \left\{u^{\prime E(x, y)}\left(g_{i}(b)\right): b \in A^{R} \cup\{x, y\} \quad \text { such that } \quad g_{i}(b) \geq g_{i}(a)\right\}
$$

Note that $\mathbf{u}^{\mathbf{E}(\mathbf{x}, \mathbf{y})}$ and $\mathbf{u}^{\prime \mathbf{A}}$ satisfies conditions 1-4 of above proposition 4.1 and, as for hypothesis $\mathbf{u}^{\prime \mathbf{E}(\mathbf{x}, \mathbf{y})}$ satisfies constraints $E(x, y)$, then $\mathbf{u}^{\prime \mathbf{A}}$ satisfies constraints $E^{A}$. Calculating $U(x)-U(y)$ in correspondence of $\mathbf{u}^{\mathbf{A}}$ we obtain $U(x)-U(y)=d(x, y)$. Observe that in $\mathbf{u}^{\mathbf{A}} U(x)-U(y)=$ $d^{A}(x, y)$. But $d^{A}(x, y)>d(x, y)$ and therefore it is false that $U(x)-U(y)$ reaches its minimum in $E^{A}$ in $\mathbf{u}^{\mathbf{A}}$. Thus if $d^{A}(x, y)>d(x, y)$, then the minimum of $U(x)-U(y)$ in $E^{A}$ would not be $d^{A}(x, y)$, and this is absurd.

- $d^{A}(x, y)<d(x, y)$ : consider $\mathbf{u}^{\mathbf{E}(\mathbf{x}, \mathbf{y})}$ such that for all $i=\ldots n$

- $u^{E(x, y)}\left(\alpha_{i}\right)=u^{A}\left(\alpha_{i}\right)=0, u^{E(x, y)}\left(\beta_{i}\right)=u^{A}\left(\beta_{i}\right)$,

- for all $a \in A^{R} \cup\{x, y\}$

$$
u^{E(x, y)}\left(g_{i}(a)\right)=u^{A}\left(g_{i}(a)\right)
$$

Note that $\mathbf{u}^{\mathbf{E}(\mathbf{x}, \mathbf{y})}$ and $\mathbf{u}^{\mathbf{A}}$ satisfies conditions 1-4 of above proposition 4.1 and, as for hypothesis $\mathbf{u}^{\mathbf{A}}$ satisfies constraints $E^{A}$, then $\mathbf{u}^{\mathbf{E}(\mathbf{x}, \mathbf{y})}$ satisfies constraints $E(x, y)$.

Calculating $U(x)-U(y)$ in correspondence of $\mathbf{u}^{\mathbf{E}(\mathbf{x}, \mathbf{y})}$ we obtain $U(x)-U(y)=d^{A}(x, y)$. Observe that in $\mathbf{u}^{\mathbf{E}(\mathbf{x}, \mathbf{y})} U(x)-U(y)=d(x, y)$. But $d^{A}(x, y)<d(x, y)$ and therefore it is false that $U(x)-U(y)$ reaches its minimum in $E(x, y)$ in $\mathbf{u}^{\mathbf{E}(\mathbf{x}, \mathbf{y})}$. Thus if $d^{A}(x, y)<d(x, y)$, then the minimum of $U(x)-U(y)$ in $E(x, y)$ would not be $d(x, y)$, and this is absurd.

As $d^{A}(x, y)>d(x, y)$ and $d^{A}(x, y)<d(x, y)$ are both absurd then $d^{A}(x, y)=d(x, y)$. An analogous proof holds for $D^{A}(x, y)=D(x, y)$.

Now we are ready to make clear the semantics of the two relations $\succsim^{S}$ and $\succsim^{W}$ by giving hereafter some of their properties.

Proposition 4.3. $\succsim^{W} \supseteq \succsim^{S}$

Proof: If for $x, y \in A$, we have that $x \succsim^{S} y$, then $d(x, y) \geq 0$. As

$$
D(x, y)=\operatorname{Max}_{\text {s.t.E(x,y) }}\{U(x)-U(y)\} \geq \operatorname{Min}_{\text {s.t.E }(x, y)}\{U(x)-U(y)\}=d(x, y) \geq 0,
$$

then $x \succsim^{W} y$.

In more intuitive terms, on the basis of the semantics of $\succsim^{W}$ and $\succsim^{S}$, proposition 4.3 says that if $U(x) \geq U(y)$ for all compatible value functions $U$, i.e. $x \succsim^{S} y$, then there is at least one value function $U^{\prime}$ such that $U^{\prime}(x) \geq U^{\prime}(y), x \succsim^{W} y$. 
Proposition 4.4. $\succsim^{S}$ is a partial preorder (i.e, reflexive and transitive)

Proof: For all $x \in A U(x)=U(x)$ such that $\operatorname{Min}_{\text {s.t.E(x,y) }}\{U(x)-U(x)\}=d(x, x) \geq 0$ and thus $x \succsim^{S} x$, i.e. $\succsim^{S}$ is reflexive.

Suppose $\succsim^{S}$ is not transitive, i.e, $\exists x, y, z \in A$ such that $x \succsim^{S} y$ and $y \succsim^{S} y$ but not $x \succsim^{S} z$. This means $d(x, y) \geq 0, d(y, z) \geq 0$ and $d(y, z)<0$ which, for above proposition 4.2 , gives $d^{A}(x, y) \geq 0$, $d^{A}(y, z) \geq 0$ and $d^{A}(y, z)<0$. This means that $U(x)-U(z)$ takes its minimum value subject to constraints $E^{A}$ in $\mathbf{u}^{* \mathbf{A}}$ to which corresponds a value function denoted by $U^{*}$ such that $U^{*}(x)<U^{*}(z)$. But as we are supposing that $d^{A}(x, y)=\min _{\text {s.t. } E^{A}} U(x)-U(y) \geq 0$ and $d^{A}(y, z)=\min _{\text {s.t. } E^{A}} U(y)-$ $U(z) \geq 0$, then $U(x) \geq U(y)$ and $U(y) \geq U(z)$ for all the compatible value functions corresponding to some $\mathbf{u}^{\mathbf{A}}$ satisfying constraints $E^{A}$. Therefore we have also $U^{*}(x) \geq U^{*}(y)$ and $U^{*}(y) \geq U^{*}(z)$ for the specific value function $U^{*}$. Consequently, one should get: $U^{*}(x) \geq U^{*}(y) \geq U^{*}(z)>U^{*}(x)$, which is absurd and therefore $\succsim^{S}$ is transitive.

Proposition 4.5. $\succsim^{W}$ is strongly complete (i.e, $\forall x, y \in A, x \succsim^{W}$ y or $y \succsim^{W} x$ )

Proof: Observe that

$$
\text { not } x \succsim^{W} y \Leftrightarrow D(x, y)<0 \Leftrightarrow \operatorname{Max}_{\text {s.t.E }(x, y)}\{U(x)-U(y)\}<0
$$

but

$$
\operatorname{Max}_{\text {s.t.E }(x, y)}\{U(x)-U(y)\}=-\operatorname{Min}_{\text {s.t.E }(x, y)}\{U(y)-U(x)\}
$$

and thus, after noting that $E(x, y)=E(y, x)$

$$
\operatorname{Max}_{\text {s.t.E(x,y) }}\{U(x)-U(y)\}<0 \Leftrightarrow \operatorname{Min}_{\text {s.t.E }(y, x)}\{U(y)-U(x)\}>0 .
$$

As

$$
\operatorname{Max}_{\text {s.t.E }(y, x)}\{U(y)-U(x)\} \geq \operatorname{Min}_{\text {s.t.E(y,x) }}\{U(y)-U(x)\}
$$

from $(i)$ and $(i i)$ we obtain

$$
D(y, x)=\operatorname{Max}_{\text {s.t.E }(y, x)}\{U(y)-U(x)\}>0 \Rightarrow y \succsim^{W} x
$$

In conclusion, from $(i),(i i)$ and $(i i i)$ we get

$$
\text { not } x \succsim^{W} y \Rightarrow y \succsim^{W} x
$$

which is the thesis.

We can express the proof of proposition 4.5 in more intuitive terms as follows. Consider any compatible value function $U$. For each pair $x, y \in A$, it holds $U(x) \geq U(y)$ or $U(y) \geq U(x)$, i.e., $x \succsim^{W} y$ or $y \succsim^{W} x$.

Observe that $\succsim^{W}$ is not necessarily transitive. In fact it is possible that for $x, y, z \in A, x \succsim^{W} y$, $y \succsim^{W} z$ but not $x \succsim^{W} z$. This can happen because there is no relation between

$$
\begin{aligned}
& D(x, y)=\operatorname{Max}_{\text {s.t.E }(x, y)}\{U(x)-U(y)\}, \\
& D(y, z)=\operatorname{Max}_{\text {s.t.E }(y, z)}\{U(y)-U(z)\},
\end{aligned}
$$

and

$$
D(x, z)=\operatorname{Max}_{\text {s.t.E }(x, z)}\{U(x)-U(z)\} .
$$

In more intuitive terms, this means that there could exist one compatible value function $U$ such that $U(x) \geq U(y)$ and one compatible value function $U^{\prime}$ such that $U^{\prime}(y) \geq U^{\prime}(z)$, and nevertheless there could be no value function $U^{\prime \prime}$ such that $U^{\prime \prime}(x) \geq U^{\prime \prime}(z)$.

Let us now explain how to exploit the two weak preference relations $\succsim^{S}$ and $\succsim^{W}$ : 
1. We get the strong ranking defined by:

- preference: $x \succ^{S} y \Leftrightarrow x \succsim^{S} y$ and not $y \succsim^{S} x$

- indifference: $x \sim^{S} y \Leftrightarrow x \succsim^{S} y$ and $y \succsim^{S} x$

- incomparability: $x ?^{S} y \Leftrightarrow$ not $x \succsim^{S} y$ and not $y \succsim^{S} x$

2. We get the weak ranking defined by:

- preference: $x \succ^{W} y \Leftrightarrow x \succsim^{W} y$ and not $y \succsim^{W} x$

- indifference: $x \sim^{W} y \Leftrightarrow x \succsim^{W} y$ and $y \succsim^{W} x$

What are the relations between the $\succsim^{W}$ and $\succsim^{S}$ ? Following proposition 4.6 gives a technical result useful to discuss this point.

Proposition 4.6. The following equivalences hold: for all $x, y \in A$

$$
\begin{aligned}
& d(x, y) \geq 0 \Leftrightarrow D(y, x) \leq 0 \\
& D(x, y) \geq 0 \Leftrightarrow d(y, x) \leq 0 \\
& d(x, y)=0 \Leftrightarrow D(y, x)=0
\end{aligned}
$$

Proof: Let us remark that

$d(x, y)=\operatorname{Min}_{\text {s.t.E }(x, y)}\{U(x)-U(y)\}=-\operatorname{Max}_{\text {s.t. } E(y, x)}\{U(y)-U(x)\}=-D(y, x)$

from which we obtain the thesis.

Note that it is impossible to infer one ranking from the other. One could think that $\succsim^{S}$ and $\succsim^{W}$ could be dual, i.e., for all $x, y \in A$,

$$
x \succsim^{S} y \Leftrightarrow \operatorname{not} y \succsim^{W} x
$$

or, equivalently,

$$
\text { not } x \succsim^{S} y \Leftrightarrow y \succsim^{W} x \text {. }
$$

Taking into account the semantics of $\succsim^{S}$ and $\succsim^{W}$, this would mean that for all compatible value functions $U(x) \geq U(y)$ if and only if there would be no value function for which $U(y) \geq U(x)$, or, equivalently, there would be no value function for which $U(x) \geq U(y)$ if and only if for all compatible value functions $U(y) \geq U(x)$.

In fact, we have that

$$
\text { not } x \succsim^{W} y \Rightarrow y \succsim^{S} x
$$

because, for above proposition 4.6 , one can get

$$
\operatorname{not} x \succsim^{W} y \Leftrightarrow D(x, y)<0 \Rightarrow d(y, x) \geq 0 \Leftrightarrow y \succsim^{S} x \text {. }
$$

However we have not that

$$
\text { not } x \succsim^{W} y \Leftarrow y \succsim^{S} x
$$

because, for above proposition 4.6, one can get only

$$
D(x, y) \leq 0 \Leftarrow d(y, x) \geq 0 \Leftrightarrow y \succsim^{S} x,
$$

and not

$$
\operatorname{not} x \succsim^{W} y \Leftrightarrow D(x, y)<0 \Leftarrow d(y, x) \geq 0 \Leftrightarrow y \succsim^{S} x
$$


which is false in case $D(x, y)=d(x, y)=0$ (i.e. in case $U(x)=U(y)$ for all compatible value functions $U)$. Therefore above $(i)$ is false.

Analogously, we have

$$
\text { not } x \succsim^{S} y \Rightarrow y \succsim^{W} x
$$

but we have not

$$
\operatorname{not} x \succsim^{S} y \Leftarrow y \succsim^{W} x
$$

Therefore also above $(\mathrm{ii})$ is false.

In more intuitive terms, taking into account the semantics of $\succsim^{W}$ and $\succsim^{S},(i)$ is false because we can have that for all compatible value functions $U(x)=U(y)$, such that it is true that for all compatible value functions $U(x) \geq U(y)$ (i.e. $x \succsim^{S} y$ is true) but it is false that there is no compatible value function such that $U(y) \geq U(x)$ (i.e. "not $x \succsim^{S} y$ " is false). Analogously, one can see that in case $U(x)=U(y)$ for all compatible value functions, also $(i i)$ is false.

Observe also that the fact that $\succsim^{W}$ and $\succsim^{S}$ are not related each other, does not imply that to define both of them, and consequently the related weak and strong ranking, one needs to calculate both $d(x, y)$ and $D(x, y), x, y \in A$. In fact, according to proposition 4.6, the strong ranking can be computed using either $d(x, y)$ or $D(x, y)$ as shown in Table 1 and 2. A similar remark concerns the weak ranking which can be computed using either $d(x, y)$ or $D(x, y)$, as shown in Table 3 and 4 .

\begin{tabular}{|c|c|c|c|c|}
\cline { 4 - 5 } \multicolumn{2}{c|}{} & \multicolumn{2}{c|}{$y \succsim^{S} x$} & not $y \succsim^{S} x$ \\
\cline { 4 - 5 } \multicolumn{2}{c|}{} & $d(y, x)>0$ & $d(y, x)=0$ & $d(y, x)<0$ \\
\hline \multirow{2}{*}{$x \succsim^{S} y$} & $d(x, y)>0$ & & & $x \succ^{S} y$ \\
\cline { 4 - 5 } & $d(x, y)=0$ & & $x \sim^{S} y$ & $x \succ^{S} y$ \\
\hline not $x \succsim^{S} y$ & $d(x, y)<0$ & $y \succ^{S} x$ & $y \succ^{S} x$ & $x ? y$ \\
\hline
\end{tabular}

Table 1: Strong ranking in terms of $d(x, y)$

\begin{tabular}{|c|c||c|c|c|}
\cline { 3 - 5 } \multicolumn{2}{c|}{} & $\operatorname{not} x \succsim^{S} y$ & \multicolumn{2}{|c|}{$x \succsim^{S} y$} \\
\cline { 2 - 5 } \multicolumn{2}{c|}{} & $D(y, x)>0$ & $D(y, x)=0$ & $D(y, x)<0$ \\
\hline not $y \succsim^{S} x$ & $D(x, y)>0$ & $x ? y$ & $x \succ^{S} y$ & $x \succ^{S} y$ \\
\hline \multirow{2}{*}{$y \succsim^{S} x$} & $D(x, y)=0$ & $y \succ^{S} x$ & $x \sim^{S} y$ & \\
\cline { 2 - 4 } & $D(x, y)<0$ & $y \succ^{S} x$ & & \\
\hline
\end{tabular}

Table 2: Strong ranking in terms of $D(x, y)$

Remark 4.1. In the absence of any pairwise comparison of reference alternatives, the strongly established weak preference relation $\succsim^{S}$ boils down to the weak dominance relation $\Delta$ in $A(a \Delta b$ iff $\left.g_{i}(a) \geq g_{i}(b), i=1, . ., n\right)$. Each pairwise comparison provided by the DM contributes to enrich $\succsim^{S}$, i.e., it makes the relation $\succsim^{S}$ true for at least one more pair of alternatives.

Remark 4.2. In the absence of any pairwise comparison of reference alternatives, the weakly established weak preference relation $\succsim^{W}$ is a complete relation such that for any pair $(a, b) \in A \times A$ 


\begin{tabular}{|c|c|c|c|c|}
\cline { 4 - 5 } \multicolumn{2}{c|}{} & \multicolumn{2}{c|}{$y \succsim^{W} x$} & not $y \succsim^{W} x$ \\
\cline { 4 - 5 } \multicolumn{2}{c|}{} & $d(y, x)>0$ & $d(y, x)=0$ & $d(y, x)<0$ \\
\hline \multirow{2}{*}{$x \succsim^{W} y$} & $d(x, y)>0$ & & & $x \succ^{W} y$ \\
\cline { 2 - 5 } & $d(x, y)=0$ & & $x \sim^{W} y$ & $x \sim^{W} y$ \\
\hline not $x \succsim^{W} y$ & $d(x, y)<0$ & $y \succ^{W} x$ & $x \sim^{W} y$ & $x \sim^{W} y$ \\
\hline
\end{tabular}

Table 3: Weak ranking in terms of $d(x, y)$

\begin{tabular}{|c|c||c|c|c|}
\cline { 3 - 5 } \multicolumn{1}{c|}{} & $\operatorname{not} x \succsim^{W} y$ & \multicolumn{2}{|c|}{$x \succsim^{W} y$} \\
\cline { 3 - 5 } \multicolumn{1}{c|}{} & $D(y, x)>0$ & $D(y, x)=0$ & $D(y, x)<0$ \\
\hline not $y \succsim^{W} x$ & $D(x, y)>0$ & $x \sim^{W} y$ & $x \sim^{W} y$ & $x \succ^{W} y$ \\
\hline \multirow{2}{*}{$y \succsim^{W} x$} & $D(x, y)=0$ & $x \sim^{W} y$ & $x \sim^{W} y$ & \\
\cline { 3 - 5 } & $D(x, y)<0$ & $y \succ^{W} x$ & & \\
\hline
\end{tabular}

Table 4: Weak ranking in terms of $D(x, y)$

- $a \succsim^{W} b$ and $b \succsim^{W} a \Leftrightarrow\{[\operatorname{not}(a \Delta b)$ and $\operatorname{not}(b \Delta a)]$ or $(a \Delta b$ and $b \Delta a)\}$

- $a \succsim^{W} b$ and $\operatorname{not}\left(b \succsim^{W} a\right) \Leftrightarrow(a \Delta b$ and not $b \Delta a)$

Each pairwise comparison provided by the DM contributes to impoverish $\succsim^{W}$, i.e., it makes the relation $\succsim^{W}$ false for at least one more pair of alternatives.

Let us now consider the case where the polyhedron generated by $E^{A^{R}}$ of constraints is empty. Let us remember that for proposition 4.1, in this case also the polyhedrons generated by $E(x, y)$, for all $x, y \in A$, and $E^{A}$ are empty. Such case may occur in one of the following situations:

- the preferences of the DM do not match the additive model;

- the DM may have made an error in his/her statements; for example stating that $a \succ b$ while $b$ dominates $a$;

- the statements provided the DM are in conflict because of his/her preferences are unstable, some hidden criteria are taken into account, ...

In such case, the DM may want either to pursue the analysis with such an "inconsistency" (the polyhedron generated by $E^{A^{R}}$ is empty) or to identify its reasons in order to remove it, and therefore to define a new $B^{\prime R}$ whose corresponding corresponding $E^{\prime A^{R}}$ generates a non empty polyhedron.

Let us consider the two cases.

\section{Analysis with inconsistency}

If the DM wants to pursue the analysis with the inconsistency he/she has to accept that some of his/her pairwise comparisons of reference alternatives will not be reproduced by any utility function. Note that, from a formal viewpoint, if the polyhedron generated by $E^{A^{R}}$ is empty, then $\succsim^{S}$ and $\succsim^{W}$ cannot be determined according to the above definitions 4.1 and 4.2 which require that the polyhedron of compatible value function is not empty as a prerequisite. Thus the acceptance of the inconsistency means to mantain the set of exemplary pairwise comparisons $B^{R}$, computing $d(x, y)$ and $D(x, y)$ in a new set of constraints $E^{A^{R}}$ that "minimally" differs from the original $E^{A^{R}}$. The most straightforward solution is to replace the constraints

$$
\sigma^{+}(a)=0, \sigma^{-}(a)=0, a \in A^{R}
$$


with the constraint

$$
\sum_{a \in A^{R}}\left(\sigma^{+}(a)+\sigma^{-}(a)\right) \leq \delta,
$$

where $\delta>0$ corresponds to an acceptable total error such that the resulting new set of constraints $E^{\prime A^{R}}$ is not empty.

On the basis of $E^{\prime A^{R}}$, for any pair $(x, y) \in A$, the set of constraints $E^{\prime}(x, y)$ can be built as the union of the constraints in $E^{\prime A^{R}}$ with the constraints relative to the break points relative to alternatives $x, y \notin A^{R}$. Thus preference relation $\succsim^{\prime S}$ and $\succsim^{\prime W}$ can be defined according to the above definitions 4.1. and 4.2 with the difference that $d(x, y)$ and $D(x, y)$ are computed minimizing and maximizing $U(x)-U(y)$ on $E^{\prime}(x, y)$ rather than on $E(x, y)$.

Obviously, the strong and weak rankings resulting from these computations will not fully restore the provided pairwise comparisons, i.e. there is at least one pair $(x, y) \in B^{R}$ such that

- $x \succsim y$, but it is false that for all the compatible value functions $U(x) \geq U(y)$ (thus there is also a compatible value function such that $U(x)<U(y)$ and therefore not $x \succsim^{\prime S} y$ ), or

- $x \succ y$, but it is false that for all the compatible value functions $U(x)>U(y)$ (thus there is also a value function such that $U(y) \geq U(x)$ and therefore $\left.y \succsim^{\prime W} x\right)$.

Next result stated that $\succsim^{S}$ and $\succsim^{\prime W}$ mantain all the main properties of preference relations $\succsim^{S}$ and $\succsim^{W}$.

\section{Proposition 4.7.}

- $\succsim^{S} \subseteq \succsim^{\prime W}$

- $\succsim^{\prime S}$ is a complete preorder (i.e. transitive and strongly complete),

- $\succsim^{\prime W}$ is strongly complete.

Proof: Analogous to the proof of propositions 4.3, 4.4 and 4.5.

\section{Analysis removing the inconsistency}

if the DM does not want to pursue the analysis with the inconsistency, it is necessary to identify the inconsistent pairwise comparisons, so as to remove them. Remark that there may exist several sets of pairwise comparisons which, once removed, make set $E^{A^{R}}$ of constraints non-empty. Hereafter, we outline the main steps of a procedure which identifies these sets.

Recall that the pairwise comparisons of reference alternatives are represented in the ordinal regression constraints $\left(E^{A^{R}}\right)$ by linear constraints. Hence, identifying inconsistent pairwise comparisons of reference alternatives amounts at finding a minimal subset of constraints that, once removed from $E^{A^{R}}$, leads to a set of constraints generating a non-empty set polyhedron of feasible value functions. The identification procedure is to be performed iteratively since there may exist several minimal subsets of this kind.

Let associate with each pairwise comparison concerning reference alternatives $a$ and $b$ a new binary variable $v_{a, b}$. Using these binary variables, we rewrite the first two constraints of set $(E)$ as follows: 


$$
\begin{aligned}
& a \succ b \Leftrightarrow U^{\prime}(a)-U^{\prime}(b)+M v_{a, b}>0 \\
& a \sim b \Leftrightarrow\left\{\begin{array}{l}
U^{\prime}(a)-U^{\prime}(b)+M v_{a, b} \geq 0 \\
U^{\prime}(b)-U^{\prime}(a)+M v_{a, b} \geq 0
\end{array}\right.
\end{aligned}
$$

where $M$ is an arbitrary large positive number. Remark that if $v_{a, b}=1$, then the corresponding constraint is satisfied whatever the value function is, which is equivalent to deletion of this constraint. Therefore, identifying a minimal subset of inconsistent pairwise comparisons can be performed by solving the following mixed 0-1 linear program:

$$
\begin{aligned}
\text { Min } \rightarrow \quad f & =\sum_{(a, b) \in B^{R}} v_{a, b} \\
\text { s.t. } & \\
& a \succ b \Leftrightarrow U^{\prime}(a)-U^{\prime}(b)+M v_{a, b} \geq \varepsilon \\
& a \sim b \Leftrightarrow\left\{\begin{array}{l}
U^{\prime}(a)-U^{\prime}(b)+M v_{a, b} \geq 0 \\
U^{\prime}(b)-U^{\prime}(a)+M v_{a, b} \geq 0
\end{array}\right\} \forall(a, b) \in B^{R} \\
& u_{i}\left(g_{i}\left(a_{\tau_{i}(j)}\right)\right)-u_{i}\left(g_{i}\left(a_{\tau_{i}(j-1)}\right)\right) \geq 0, i=1, \ldots, n, j=1, \ldots, m \\
& u_{i}\left(g_{i}\left(a_{\tau_{i}(1)}\right)\right) \geq 0, u_{i}\left(g_{i}\left(a_{\tau_{i}(m)}\right)\right) \leq u_{i}\left(\beta_{i}\right), i=1, \ldots, n, \\
& u_{i}\left(\alpha_{i}\right)=0, i=1, \ldots, n \\
& \sum_{i=1}^{n} u_{i}\left(\beta_{i}\right)=1, \\
& \sigma^{+}(a)=0, \sigma^{-}(a)=0, \forall a \in A^{R}
\end{aligned}
$$

where $\tau_{i}$, already introduced in defining set of constraints $E^{A^{R}}$, is the permutation on the set of alternatives $A^{R}$ that reorders them according to the increasing evaluation on criterion $g_{i}$, i.e.

$$
g_{i}\left(a_{\tau_{i}(1)}\right) \leq g_{i}\left(a_{\tau_{i}(2)}\right) \leq \ldots \leq g_{i}\left(a_{\tau_{i}(m-1)}\right) \leq g_{i}\left(a_{\tau_{i}(m)}\right) .
$$

The optimal solution of (14) indicates one of the subsets of smallest cardinality being the cause of inconsistency. Alternative subsets of this kind can be found by solving (14) with additional constraint that forbids finding again the same solution. Let $f^{*}$ be the optimal value of the objective function of (14) and $v_{a, b}^{*}$ the values of the binary variables at the optimum. Let also $S_{1}=\left\{(a, b) \in B^{R}: v_{a, b}^{*}=1\right\}$. The additional constraint has then the form

$$
\sum_{(a, b) \in S_{1}} v_{a, b}<f^{*}
$$

Continuing in this way, we can identify other subsets, possibly all of them. These subsets of pairwise comparisons are to be presented to the DM as alternatives solutions for removing inconsistency. Such procedure has been described in [15]

\section{Extensions}

\subsection{Specification of pairwise comparisons with gradual confidence levels}

The UTA ${ }^{\text {GMS }}$ method presented in the previous section is intended to support the DM in an interactive process. Indeed, defining a large set of pairwise comparisons of reference alternatives can be difficult for the DM. Therefore, one way to reduce the difficulty of this task would be to permit the DM an incremental specification of pairwise comparisons. This way of proceeding allows the DM to control the evolution of the strongly and weakly established weak preference relations.

Another way of reducing the difficulty of the task is to extend the UTA ${ }^{\text {GMS }}$ method so as to account for different confidence levels assigned to pairwise comparisons. Let $B_{1}^{R} \subseteq B_{2}^{R} \subseteq \ldots \subseteq B_{s}^{R}$ be 
embedded sets of pairwise comparisons of reference alternatives. To each set of pairwise comparison $B_{t}^{R}, t=1, \ldots s$ corresponds a set of constraints $E_{t}^{A^{R}}$ generating polyhedrons of compatible value functions $P_{t}^{A^{R}}$ which are embedded in the inverse order of the related sets $B_{t}^{R}$, i.e. $P_{1}^{A^{R}} \supseteq P_{2}^{A^{R}} \supseteq$ $\ldots \supseteq P_{s}^{A^{R}}$. We suppose that $P_{s}^{A^{R}} \neq \emptyset$ and therefore, due the fact that sets $B_{t}^{R}$ are embedded, $P_{t}^{A^{R}} \neq \emptyset$, for all $t=1, \ldots, s$. If $P_{s}^{A^{R}}=\emptyset$ we consider only embedded sets until $B_{p}^{R}$ with $p=\max \left\{t: P_{t}^{A^{R}} \neq \emptyset\right\}$ and relabel $p$ as $s$. For all $x, y \in A$, set of constraints $E_{t}(x, y)$ can be obtained from the set $E_{t}^{A^{R}}$ by adjoining the constraints relative to the breakpoints of $x, y \notin A^{R}$. For each $t=1, \ldots, s$, binary preference relations $\succsim_{t}^{S}$ and $\succsim_{t}^{W}$ can be defined as follows.

Definition 5.1. $\succsim_{t}^{S}$ (strongly established weak preference relation of level $t, t=1, \ldots, s$ ) is defined as follows: for all $x, y \in A$

$$
x \succsim_{t}^{S} y \Leftrightarrow d_{t}(x, y) \geq 0
$$

where:

$$
\begin{aligned}
d_{t}(x, y)= & \operatorname{Min}\{U(x)-U(y)\} \\
& \text { s.t. set } E_{t}(x, y) \text { of constraints }
\end{aligned}
$$

Definition 5.2. $\succsim_{t}^{W}$ (weakly established weak preference relation of level $\left.t, t=1, \ldots, s\right)$ is defined as follows: for all $x, y \in A$

where :

$$
x \succsim_{t}^{W} y \Leftrightarrow D_{t}(x, y) \geq 0
$$

$$
\begin{aligned}
D_{t}(x, y)= & \operatorname{Max}\{U(x)-U(y)\} \\
& \text { s.t. set } E_{t}(x, y) \text { of constraints }
\end{aligned}
$$

Each time we pass from $B_{t-1}^{R}$ to $B_{t}^{R}, t=1, \ldots, s-1$, we add to $E_{t-1}^{A^{R}}$, and consequently to $E_{t-1}(x, y)$, new constraints concerning pairwise comparisons from $B_{t}^{R} \backslash B_{t-1}^{R}$, thus the computations of $d_{t}(x, y)$ and $D_{t}(x, y)$, for all $x, y \in A \times A$, using (16) and (17), respectively, proceeds iteratively.

The following result states that binary preference relations $\succsim_{t}^{S}$ and $\succsim_{t}^{W}, t=1, \ldots, s$, inherit properties of $\succsim^{S}$ and $\succsim^{W}$.

\section{Proposition 5.1.}

- $\succsim_{t}^{S \subseteq \succsim_{t}^{W}}$

- $\succsim_{t}^{S}$ is a complete preorder (i.e. transitive and strongly complete),

- $\succsim_{t}^{W}$ is strongly complete.

Proof: Analogous to the proof of propositions 4.3, 4.4 and 4.5.

A specific property of binary preference relations $\succsim_{t}^{S}$ and $\succsim_{t}^{W}, t=1, \ldots, s$, is that, due to the fact that $P_{t-1}^{A^{R}} \supseteq P_{t}^{A^{R}}, t=2, \ldots, k$, they are nested partial preorders, i.e., $\succsim_{t-1}^{S} \supseteq \succsim_{t}^{S}$ and $\succsim_{t}^{W} \supseteq_{\tau_{t-1}}^{W}$, $t=2, \ldots, s$.

Let $\lambda_{t}$ be the confidence level assigned to pairwise comparisons from $B_{t}^{R} \backslash B_{t-1}^{R}, B_{0}^{R}=\emptyset, t=1, \ldots, s$, $0<\lambda_{1}<\lambda_{2}<\ldots<\lambda_{s}=1, t=1, \ldots, s$.

Using sets $B_{1}^{R}, \ldots B_{s}^{R}$ and corresponding $\lambda_{1}, \lambda_{2}, \ldots, \lambda_{s}$, a valued binary preference relations $R^{S}$ : $A \times A \rightarrow[0,1]$ (more precisely $R^{S}: A \times A \rightarrow\left\{0, \lambda_{1}, \lambda_{2}, \ldots, \lambda_{s}\right\}$ ) can be build as follows: for all $x, y \in A$ 
- if there exists one $t=1, \ldots, s$ such that $x \succsim_{t}^{S} y$, then

$$
R^{S}(x, y)=\max \left\{\lambda_{t}, t=1, \ldots, s: \text { such that } x \succsim_{t}^{S} y\right\}
$$

- if not $x \succsim_{t}^{S} y$ for all $t=1, \ldots, s$, then $R^{S}(x, y)=0$.

Analogously, a valued binary preference relations $R^{W}: A \times A \rightarrow[0,1]$ (more precisely $R^{W}:$ $\left.A \times A \rightarrow\left\{\lambda_{1}, \lambda_{2}, \ldots, \lambda_{s}, 0\right\}\right)$ can be build as follows: for all $x, y \in A$

- if there exists one $t=1, \ldots, s$ such that $x \succsim_{t}^{W} y$, then

$$
R^{W}(x, y)=\max \left\{\lambda_{t}, t=1, \ldots, s: \text { such that } x \succsim_{t}^{W} y\right\}
$$

- if not $x \succsim_{t}^{W} y$ for all $t=1, \ldots, s$, then $R^{W}(x, y)=0$.

It is interesting to investigate the properties of valued binary relations $R^{S}$ and $R^{W}$. Let us remember that a valued binary relation $R$ defined on a set $X$, i.e. $R: X \times X \rightarrow[0,1]$, is

- reflexive, if for all $x \in X, R(x, x)=1$,

- min-transitive, if for all $x, y, z \in X$

$$
\min (R(x, y), R(y, z)) \leq R(x, z)
$$

- strongly complete, if for all $x, y \in X$

$$
\max (R(x, y), R(y, x))=1
$$

A valued binary relation reflexive and min-transitive is defined fuzzy preorders.

Proposition 5.2. Valued binary relation $R^{S}$ is reflexive and min-transitive, and therefore it is a fuzzy preorder. Valued binary relation $R^{W}$ is strongly complete.

Proof: For all $x \in A$ and for all value functions $U$, therefore also for the value functions compatible with $E_{s}(x, y), U(x)=U(x)$. Thus $\forall x \in A d_{s}(x, x)=\operatorname{Min}_{\text {s.t. } E_{s}(x, x)}\{U(x)-U(x)\}=0$ and in conclusion $d_{s}(x, x) \geq 0$, which implies $x \succsim_{s}^{S} x$ and therefore $R^{S}(x, x)=1$, i.e. $R^{S}$ is reflexive.

For all $x, y, z \in X$, two cases are possible:

- a) $\min \left(R^{S}(x, y), R^{S}(y, z)\right)=0$,

- b) $\min \left(R^{S}(x, y), R^{S}(y, z)\right)>0$.

Considering that anyway $R^{S}(x, z) \geq 0$, in case a), clearly,

$$
R^{S}(x, z) \geq \min \left(R^{S}(x, y), R^{S}(y, z)\right) .
$$

In case b), for the definition of $R^{S}$, we have that

$$
\begin{gathered}
\min \left(R^{S}(x, y), R^{S}(y, z)\right)= \\
=\min \left\{\max \left\{\lambda_{t}, t=1, \ldots, s: \text { such that } x \succsim_{t}^{S} y\right\}, \max \left\{\lambda_{t}, t=1, \ldots, s: \text { such that } y \succsim_{t}^{S} z\right\}\right\}= \\
=\max \left\{\lambda_{t}, t=1, \ldots, s: \text { such that } x \succsim_{t}^{S} y \text { and } y \succsim_{t}^{S} z\right\} .
\end{gathered}
$$


Thus if

$$
\min \left(R^{S}(x, y), R^{S}(y, z)\right)=\lambda_{r}
$$

then, for definition of $R^{S}$ and for proposition 4.2, $d_{r}(x, y)=\min _{\text {s.t. } E_{r}^{A}}\{U(x)-U(y)\} \geq 0$ and $d_{r}(y, z)=\min _{\text {s.t. } E_{r}^{A}}\{U(y)-U(z)\} \geq 0$. This means that $U(x) \geq U(y)$ and $U(y) \geq U(z)$ for all the value functions compatible with $B_{r}^{R}$, i.e. for all value functions $U$ corresponding to some $\mathbf{u}^{\mathbf{A}}$ satisfying constraints $E_{r}^{A}$. Thus for all these value functions we have $U(x) \geq U(y) \geq U(z)$, such that $d_{r}(x, z)=\min _{\text {s.t. } E_{r}^{A}}\{U(x)-U(z)\} \geq 0$ and consequently $x \succsim_{r}^{S} y$. This implies that

$$
\max \left\{\lambda_{t}, t=1, \ldots, s: \text { such that } x \succsim_{t}^{S} z\right\} \geq \lambda_{r} .
$$

and remembering the definition of $R^{S}$ and (ii) we obtain again $(i)$. This means that valued binary relation $R^{S}$ is min-transitive.

Observe that $R^{W}(x, y)<1$ means that

$$
D_{s}(x, y)<0 \quad(i i i)
$$

From definition of $D_{s}(x, y),($ iii $)$ gives

$$
\operatorname{Max}_{\text {s.t. } E_{s}(x, y)}\{U(x)-U(y)\}<0 \quad(i v)
$$

Remember that $E_{s}(x, y)=E_{s}(y, x)$ and observe that

$$
\operatorname{Max}_{\text {s.t. } E_{s}(y, x)}\{U(y)-U(x)\}=-\operatorname{Min}_{\text {s.t. } E_{s}(x, y)}\{U(x)-U(y)\}
$$

From $(i v),(v)$ and defintition of $D_{s}(y, x)$ we get

$$
\begin{gathered}
D_{s}(y, x)=\operatorname{Max}_{\text {s.t. } E_{s}(y, x)}\{U(y)-U(x)\} \geq \\
\geq \operatorname{Min}_{\text {s.t. } E_{s}(y, x)}\{U(y)-U(x)\}=-\operatorname{Max}_{\text {s.t. } E_{s}(x, y)}\{U(x)-U(y)\}>0
\end{gathered}
$$

which, for the definition of valued preference relation $R^{W}$, gives $R^{W}(y, x)=1$. Thus we proved that, if $R^{W}(x, y)<1$, then $R^{W}(y, x)=1$, i.e.

$$
\max \left(R^{W}(x, y), R^{W}(y, x)\right)=1
$$

which is the thesis.

\subsection{Accounting for ordinal intensity of preference}

Another preference information that can be provided by the DM concerns the intensity of preference among two pairs of reference alternatives. Given two pairs of alternatives $(a, b) \in B^{R}$ and $(c, d) \in B^{R}$ such that $a \succ b$ and $c \succ d$, the DM can state: " $a$ is preferred to $b$ at least as much as $c$ is preferred to $d "$. Such statement means that for all compatible value functions $U$ :

$$
U(a)-U(b)>U(c)-U(d)
$$

To account for the above preference information, it is sufficient to include condition (18) in set $\left.E^{A^{R}}\right)$ of constraints. Of course, consequently, condition (18) will be included in sets of constraints $E(x, y)$ for all $x, y \in A$ and in set of constraints $E^{A}$.

Conversely, $\forall x, y, w, z \in A$, it is possible to check whether or not condition

$$
U(x)-U(y)>U(w)-U(z)
$$

hold for all compatible value functions $U$.

In order to do so, it is sufficient to check the feasibility of constraints $E(x, y)$ and (19). Such information may enrich the DM's knowledge her preferences. 


\section{Illustrative example}

In this section, we illustrate how a decision aiding process can be supported by the UTA GMS method. We consider the following hypothetical decision problem. AGRITEC is a medium sized firm (350 persons approx.) producing technical products for agriculture. The C.E.O., $\mathrm{M}^{\mathrm{r}}$ Becault, intends to double the production and multiply exports by 4 within 5 years. Therefore, he wants to hire a new international sales manager. A recruitment agency has interviewed 17 potential candidates which have been evaluated on 3 criteria (sales management experience, international experience, human qualities) evaluated on a $[0,100]$ scale. The evaluations of candidates are provided in Table 5. Without any further information, the computed partial preorder $\succsim_{0}^{S}$ corresponds to the strict dominance relation on the set of alternative (See Figure 2).

\begin{tabular}{|l|c|c|c|}
\cline { 2 - 4 } \multicolumn{1}{c|}{} & Crit 1 & Crit 2 & Crit 3 \\
\hline Alexievich & 4 & 16 & 63 \\
\hline Bassama & 28 & 18 & 28 \\
\hline Calvet & 26 & 40 & 44 \\
\hline Dubois & 2 & 2 & 68 \\
\hline El Mrabat & 18 & 17 & 14 \\
\hline Feeret & 35 & 62 & 25 \\
\hline Fleichman & 7 & 55 & 12 \\
\hline Fourny & 25 & 30 & 12 \\
\hline Frechet & 9 & 62 & 88 \\
\hline Martin & 0 & 24 & 73 \\
\hline Petron & 6 & 15 & 100 \\
\hline Psorgos & 16 & 9 & 0 \\
\hline Smith & 26 & 17 & 17 \\
\hline Varlot & 62 & 43 & 0 \\
\hline Yu & 1 & 32 & 64 \\
\hline
\end{tabular}

Table 5: Evaluation Table

The C.E.O. has attended 4 interviews and can express a confident judgement about theses candidates: $M^{\mathrm{r}}$ Ferret and Frechet are equally good, $\mathrm{M}^{\mathrm{rs}}$ Fourny is less acceptable and $\mathrm{M}^{\mathrm{rs}}$ Fleichman even less acceptable. Considering such initial information, the partial preorder $\succsim_{1}^{S}$ can be computed (See Figure 3).

Considering this first result, $\mathrm{M}^{\mathrm{r}}$ Becault is wishing to add further information, i.e., Ferret Frechet $\succ$ Martin $\succ$ Fourny $\sim$ El Mrabat $\succ$ Fleichman. However, as he did not attend the interview of $\mathrm{M}^{\mathrm{r}}$ El Mrabat and $\mathrm{M}^{\mathrm{r}}$ Martin, his opinion about the relative ranking of these candidates is not definitive.

It appears that the provided information is inconsistent, i.e., no additive model fits the provided ranking. The analysis of the inconsistency reveals that the statement Ferret $\sim$ Frechet is incompatible with the statement Fourny $\sim$ El Mrabat. In other words, it is necessary for $\mathrm{M}^{\mathrm{r}}$ Becault to revise one of these statements. As he did not interviewed $\mathrm{M}^{\mathrm{r}}$ El Mrabat, he decides to remove him from the reference ranking. The resulting reference ranking is compatible with an additive representation. Figure 4 represents two embedded partial preorders: 


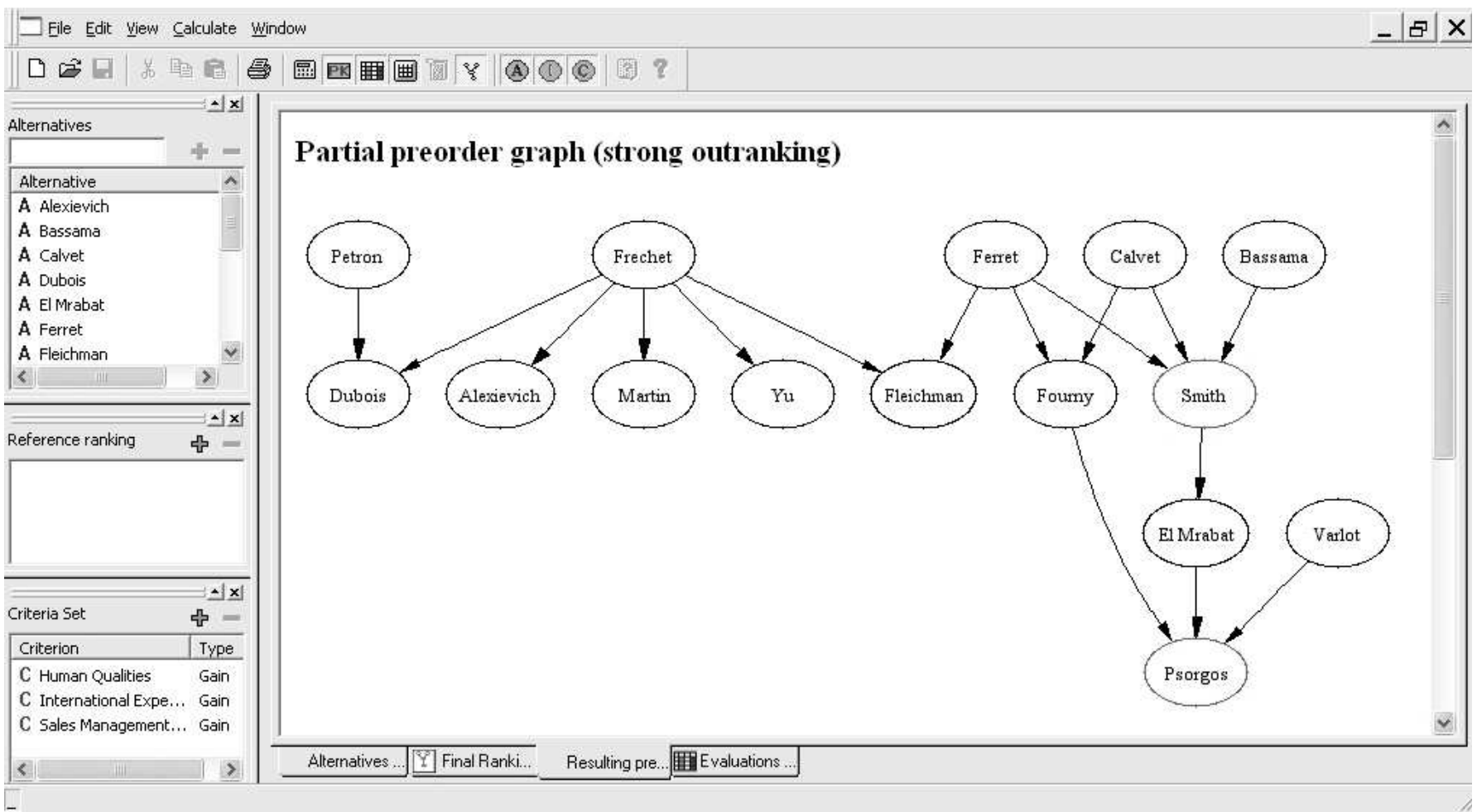

Figure 2: Partial preorder $\succsim_{0}^{S}$ corresponding to the dominance relation

- in bold arrows, the partial preorder computed from sure statements only, i.e., Ferret $\sim$ Frechet $\succ$ Fourny $\succ$ Fleichman

- in dashed arrows, the partial preorder computed from the consistent set of sure and less confident statements (sure and less confident but without considering $\mathrm{M}^{\mathrm{r}}$ El Mrabat), i.e., Ferret $\sim$ Frechet $\succ$ Martin $\succ$ Fourny $\succ$ Fleichman

The process can be pursued, $\mathrm{M}^{\mathrm{r}}$ Becault adding in iteration $t$ some new pairwise comparisons of reference alternatives, thus enriching the resulting strong partial preorder, until $\succsim_{t}^{S}$ is decisive enough for the C.E.O. to make his decision.

\section{Conclusion}

The new UTA ${ }^{\text {GMS }}$ method presented in this paper is an ordinal regression method supporting multiple criteria ranking of alternatives; it is distinguished from previous methods of this kind by the following new features:

- the method considers general additive value functions rather than piecewise linear ones,

- the final rankings are defined using all value functions compatible with the provided preference information,

- the method provides two final rankings: the strong ranking identifies "sure" preference statements while the weak ranking identifies "possible" preference statements,

- when the DM provides preference information that cannot be represented by an additive model, the method identifies which pieces of the information underly this impossibility, 


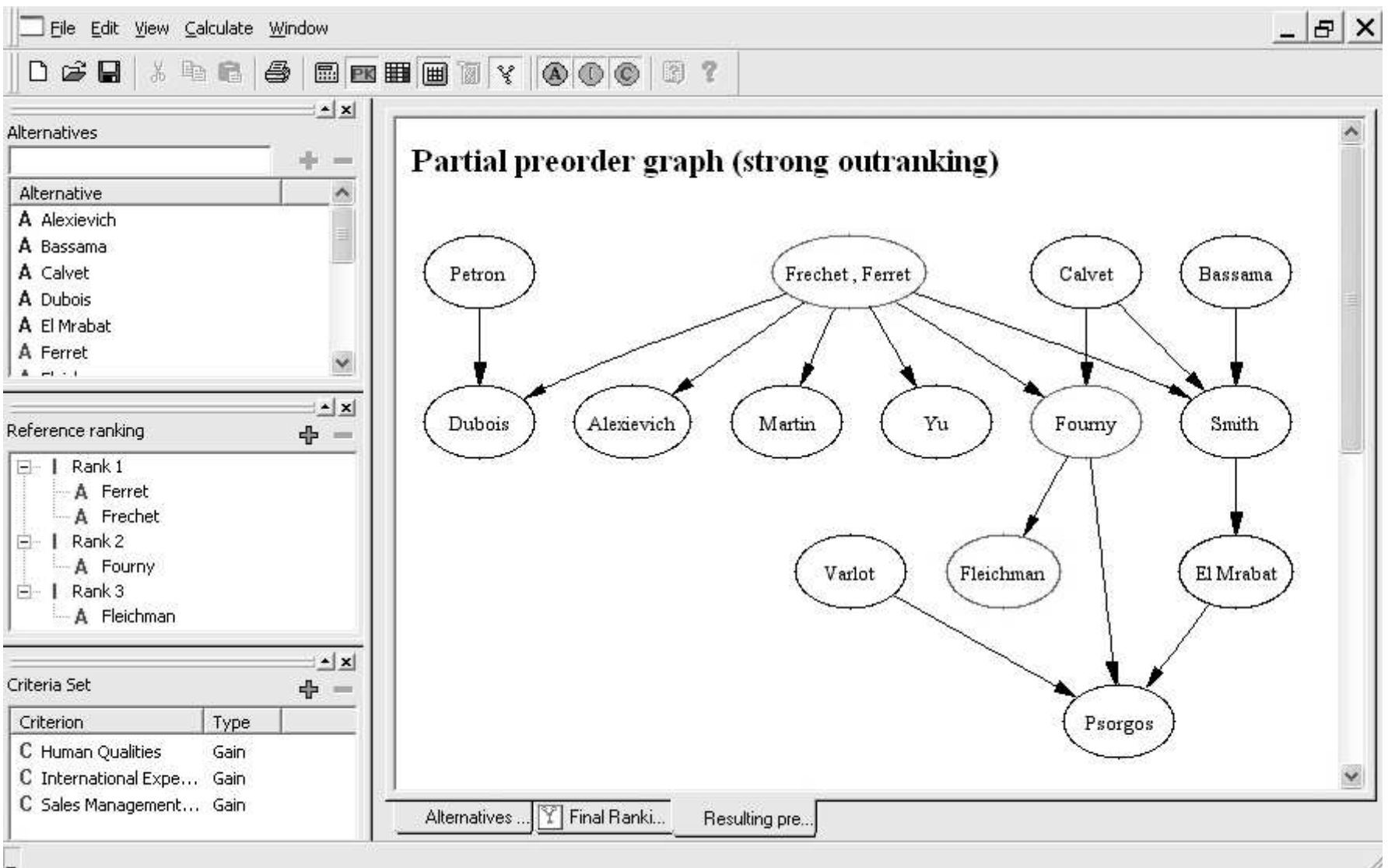

Figure 3: Partial preorder $\succsim_{1}^{S}$

- the method does not require the DM to interpret (and even look at) the marginal value functions,

- the DM can assign confidence levels to pieces of preference information,

- the method can account for ordinal intensity of preference, both a priori and a posteriori. 


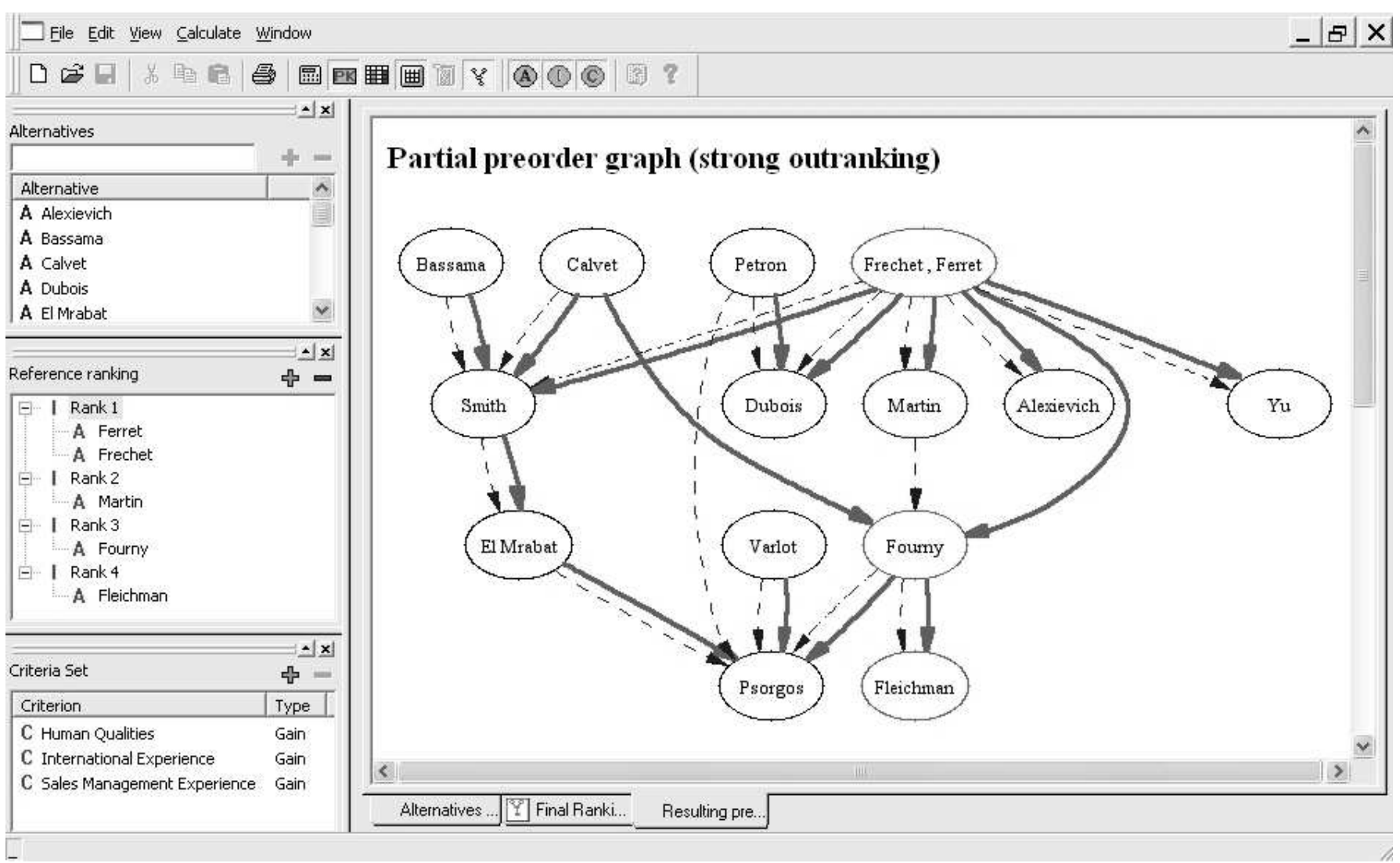

Figure 4: Nested partial preorders $\succsim_{3}^{S}$ corresponding to confidence levels

\section{References}

[1] C.A. Bana e Costa and J.C.. Vansnick. Macbeth - an interactive path towards the construction of cardinal value fonctions. International transactions in operational Research, 1:489-500, 1994.

[2] F.H. Barron. Selecting a best multiattribute alternative with partial information about attribute weights. Acta Psychologica, 80:91-103, 1992.

[3] J.M. Devaud, G. Groussaud, and E. Jacquet-Lagreze. UTADIS: Une méthode de construction de fonctions d'utilité additives rendant compte de jugements globaux. In Proceedings of the European working group on MCDA, Bochum, Germany, 1980.

[4] S. Greco, B. Matarazzo, and R. Slowinski. The use of rough sets and fuzzy sets in MCDM. In T. Gal, T. Stewart, and T. Hanne, editors, Advances in MCDM models, Algorithms, Theory, and Applications, pages 14.1-14.59. Kluwer Academic, Dordrecht, 1999.

[5] S. Greco, B. Matarazzo, and R. Slowinski. Rough sets theory for multicriteria decision analysis. European Journal of Operational Research, 129:1-47, 2001.

[6] S. Greco., B. Matarazzo, and R. Slowinski. Decision rule approach. In M. Ehrgott J. Figueira, S. Greco, editor, Multiple Criteria Decision Analysis, State of the Art Surveys, chapter 13, pages 507-561. Springer, New York, 2005.

[7] E. Jacquet-Lagrèze and Y. Siskos. Assessing a set of additive utility functions for multicriteria decision making: the UTA method. European Journal of Operational Research, 10:151-164, 1982. 
[8] C.W. Kirkwood and R.K. Sarin. Ranking with partial information: A method and an application. Operations Research, 33(1):38-48, January-February 1985.

[9] L. Kiss, J.M. Martel, and R. Nadeau. ELECCALC - an interactive software for modelling the decision maker's preferences. Decision Support Systems, 12(4-5):757-777, November 1994.

[10] M. Koksalan and V. Taner. An approach for finding the most preferred alternative in the presence of multiple criteria. European Journal of Operational Research, 60(1):53-60, 1992.

[11] M. Koksalan and C. Ulu. An interactive approach for placing alternatives in preference classes. European Journal of Operational Research, 144:429-439, 2003.

[12] K.S. Lee, K.S. Park, and S.H. Kim. Dominance, potential optimality, imprecise information, and hierarchical structure in multi-criteria analysis. Computers and Operations Research, 29:12671281, 2002.

[13] J.G. March. Bounded rationality, ambiguity and the engineering of choice. Bell Journal of Economics, 9:587-608, 1978.

[14] R.S. Michalski, I. Bratko, and M. Kubat (eds.). Machine learning and datamining - Methods and applications. Wiley, New York, 1998.

[15] V. Mousseau, J. Figueira, L.C. Dias, C. Gomes da Silva, and J.N. Clímaco. Resolving inconsistencies among constraints on the parameters of an MCDA model. European Journal of Operational Research, 147(1):72-93, May 2003.

[16] V. Mousseau and R. Slowinski. Inferring an ELECTRE TRI model from assignment examples. Journal of Global Optimization, 12(2):157-174, 1998.

[17] D. Pekelman and S.K. Sen. Mathematical programming models for the determination of attribute weights. Management Science, 20(8):1217-1229, April 1974.

[18] B. Roy. Multicriteria Methodology for Decision Aiding. Kluwer Academic, Dordrecht, 1996.

[19] B. Roy and D. Bouyssou. Aide Multicritère à la Décision: Méthodes et Cas. Economica, Paris, 1993.

[20] A.A. Salo and R.P. Hamalainen. Preference ratio in multiattribute evaluation (PRIME) - elicitation and decision procedures under incomplete information. IEEE Transactions on Systems, Man and Cybertnetics: Part A, 31(6):533-545, November 2001.

[21] Y. Siskos. A way to deal with fuzzy preferences in multicriteria decision problems. European Journal of Operational Research, 10(3):314-324, 1982.

[22] Y. Siskos, E. Grigoroudis, and N.F. Matsatsinis. UTA Methods. In J. Figueira M. Ehrgott, S. Greco, editor, State of the Art in Multiple Criteria Decision Analysis. Kluwer, 2004.

[23] V. Srinivasan and A.D. Shocker. Estimating the weights for multiple attributes in a composite criterion using pairwise judgments. Psychometrika, 38(4):473-493, 1973.

[24] M. Weber. A method of multiattribute decision making with incomplete information. Management Science, 31(11):1365-1371, 1985. 